\title{
LIE GROUP REPRESENTATIONS AND HARMONIC POLYNOMIALS OF A MATRIX VARIABLE
}

\author{
BY
}

\author{
TUONG TON-THAT
}

ABSTRACT. The first part of this paper deals with problems concerning the symmetric algebra of complex-valued polynomial functions on the complex vector space of $n$ by $k$ matrices. In this context, a generalization of the socalled "classical separation of variables theorem" for the symmetric algebra is obtained.

The second part is devoted to the study of certain linear representations, on the above linear space (the symmetric algebra) and its subspaces, of the complex general linear group of order $k$ and of its subgroups, namely, the unitary group, and the real and complex special orthogonal groups. The results of the first part lead to generalizations of several well-known theorems in the theory of group representations.

The above representation, of the real special orthogonal group, which arises from the right action of this group on the underlying vector space (of the symmetric algebra) of matrices, possesses interesting properties when restricted to the Stiefel manifold. The latter is defined as the orbit (under the action of the real special orthogonal group) of the $n$ by $k$ matrix formed by the first $n$ row vectors of the canonical basis of the $k$-dimensional real Euclidean space. Thus the last part of this paper is involved with questions in harmonic analysis on this Stiefel manifold. In particular, an interesting orthogonal decomposition of the complex Hilbert space consisting of all square-integrable functions on the Stiefel manifold is also obtained.

Introduction. Let $E_{0}=\mathbf{R}^{n \times k}$ and $E=\mathbf{C}^{n \times k}$ with $k \geqslant n$. Let $G_{0}=$ $S O(k)$ and $G=S O(k, \mathrm{C})$. The group $\operatorname{GL}(n, \mathrm{C})$ operates on $E$ to the left; the group $\operatorname{GL}(k, \mathrm{C})$ and its subgroups $U(k), G$, and $G_{0}$ act linearly on $E$ to the right. When $n=1$ and $k>2$ the following theorems are well known:

\section{THEOREM 0.1. The representations of $U(k)$ and its complexification}

Received by the editors August 16, 1974.

AMS (MOS) subject classifications (1970). Primary 13F20, 22E30, 22E45; Secondary 31C05, 33A45, 43A85.

Key words and phrases. Lie group representations, harmonic polynomial functions of a matrix variable, irreducible holomorphic representations of the special orthogonal groups, Borel-Weil-Bott theory, symmetric algebras of polynomial functions, rings of differential operators, $G$-harmonic polynomials, "generalized separation of variables" theorem, theory of polynomial invariants, generalized spherical harmonics, Stiefel manifolds, Fourier transforms, generalized Hankel transforms. 
$\mathrm{GL}(k, \mathrm{C})$ obtained by right translation on $S^{j}\left(E^{*}\right)$, the space of homogeneous polynomials of degree $j$ on $\mathbf{C}^{1 \times k}$, are irreducible.

THEOREM 0.2 ("Separation of variables" theorem).

(1) If $p \in S^{j}\left(E^{*}\right)$ then $p$ can be uniquely written as:

$$
p(X)=p_{0}(X)+r(X) p_{1}(X)+\cdots+r^{i}(X) p_{i}(X)+\cdots+r^{l}(X) p_{l}(X),
$$

where $r(X)=\Sigma_{s=1}^{k} X_{s}^{2}$ and $p_{i}$ is a homogeneous harmonic polynomial of degree $j-2 i$ on $\mathbf{C}^{1 \times k}, i=0,1, \ldots, l$, and $l=[j / 2]$.

(2) The harmonic homogeneous polynomials of degree $j$ are generated by the polynomials $f$ satisfying the following defining condition: $f(X)=\left(\sum_{i=1}^{k} A_{i} X_{i}\right)^{j}$ where the $A_{i}$ are entries of a given matrix $A \in \mathrm{C}^{1 \times k}$ with $\Sigma_{i=1}^{k} A_{i}^{2}=0$.

THEOREM 0.3. For every fixed nonnegative integer $j$ the representations of $S O(k)$ and its complexification $S O(k, C)$ obtained by right translation on $H^{j}\left(E^{*}\right)$, the space of all harmonic homogeneous polynomials of degree $j$, are irreducible.

THEOREM 0.4. The restriction mapping $f \rightarrow f / S^{k-1}$ from $H^{i}\left(E^{*}\right)$ onto $H^{i}\left(S^{k-1}\right)$, the space of all spherical harmonic functions of degree $j$, is an $S O(k)$ module isomorphism.

The references to the above theorems can be found in [6, Chapter II], [24, Chapter IX] for Theorems 0.1, 0.3, 0.4; [6, Chapter II], [24, Chapter IX], [21, Chapter IV], [4, Chapter V], and [18] for Theorem 0.2.

We obtain here a generalization of the above theorems to the case $\mathbf{C}^{n \times k}$.

The main theorems.

Definitions 0.5 . Let $(U, X)$ be a topological transformation group. Let $\lambda$ be an irreducible finite dimensional representation of $U$ on a linear space $V$. A function $f: X \rightarrow V$ will be called $\lambda$-covariant if $f(u x)=\lambda(u) f(x)$ for all $(u, x)$ in $U \times X$.

Now let $U(n)$ denote the group of unitary matrices of order $n$. Let $\xi=$ $\xi\left(m_{1}, m_{2}, \ldots, m_{n}\right)$ be a character defined on the diagonal subgroup T of $U(n)$ by $\xi(d)=d_{11}^{m_{1}} d_{22}^{m_{2}} \cdots d_{n n}^{m_{n}}$ where $d \in \mathrm{T}$ and $m_{1} \geqslant m_{2} \geqslant \cdots \geqslant m_{n}$. Let $B$ be the lower triangular subgroup of $\operatorname{GL}(n, \mathrm{C})$ and define an extension of $\xi$ to $B$ (which we shall denote again by $\xi$ ) by setting $\xi(b)=b_{11}^{m_{1}} b_{22}^{m_{2}} \cdots b_{n n}^{m_{n}}$ for all $b \in B$. Let $\mathrm{Hol}(\mathrm{GL}(n, \mathrm{C}), \xi)$ denote the space of all $\xi$-covariant holomorphic functions on $\mathrm{GL}(n, \mathrm{C})$. The representation $\pi(\cdot, \xi)$ of $\mathrm{GL}(n, \mathrm{C})$ obtained by right translation on $\operatorname{Hol}(\mathrm{GL}(n, \mathrm{C}), \xi)$ will be denoted by $\rho$. Define $P(E, \rho)$ as the linear space of all $\rho$-covariant polynomial mappings from $E$ into $H o l(G L(n, \mathbf{C}), \xi)$. Let $D(\cdot, \rho)$ be the representation of $\operatorname{GL}(k, \mathrm{C})$ on $P(E, \rho)$ obtained by right translation.

THEOREM 1.3. (i) $P(E, \rho)$ is nonzero if and only if $m_{1} \geqslant m_{2} \geqslant \cdots \geqslant$ $m_{n} \geqslant 0$. 
(ii) If $P(E, \rho)$ is nonzero, then the representation $D(\cdot, \rho)$ is irreducible and its highest weight is indexed by

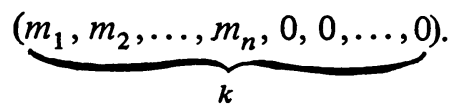

In the process of proving Theorem 1.3, we obtain the following interesting "induction in stages" type theorem.

THEOREM 1.5. If $D(\cdot, \xi)$ denotes the representation of $\operatorname{GL}(k, \mathbf{C})$ obtained by right translation on the linear space $P(E, \xi)$ of all $\xi$-covariant complex-valued polynomial functions on $E$ then, under the assumption $m_{1} \geqslant m_{2} \geqslant \cdots \geqslant m_{n} \geqslant 0$, $D(\cdot, \rho)$ and $D(\cdot, \xi)$ are equivalent; and, hence, $D(\cdot, \xi)$ is irreducible.

Notice that when $n=1, P(E, \rho)$ and $P(E, \xi)$ may both be identified to a space of homogeneous polynomials in $k$ variables of a certain fixed degree, and the notion of $\rho$-covariance as well as that of $\xi$-covariance may be thought of as generalizations of the notion of homogeneity, so that Theorems 1.3 and 1.5 do indeed generalize Theorem 0.1 . Also the study of the restriction of $D(\cdot, \rho)$ to $G$ and $G_{0}$ is equivalent to that of the restriction of $D(\cdot, \xi)$ to $G$ and $G_{0}$, respectively. This naturally leads us to investigate the symmetric algebra $S\left(E^{*}\right)$ of complex-valued polynomial functions on $E$. In this context there is a close connection between our investigation and the work of S. Helgason [12], B. Kostant [15], and H. Maass [18].

For the remainder of this investigation we must restrict ourselves to the case $k>2 n$.

Definitions 0.6. Following B. Kostant and S. Helgason (see [15] and [12] for details), we let $J\left(E^{*}\right)$ denote the subring of $S\left(E^{*}\right)$ consisting of $G$-invariant polynomial functions on $E$, and let $H\left(E^{*}\right)$ denote the subspace of $S\left(E^{*}\right)$ consisting of $G$-harmonic polynomials. We use several results in [15] to prove

THEOREM 2.5 ("Separation of variables" theorem for $\mathbf{C}^{n \times k}, k>2 n$ ).

(i) $S\left(E^{*}\right)$ is spanned by all products of the form $j h$ where $j \in J\left(E^{*}\right)$ and $h \in H\left(E^{*}\right)$; in fact, $S\left(E^{*}\right)=J\left(E^{*}\right) \otimes H\left(E^{*}\right)$ and $S\left(E^{*}\right)$ is a free $J\left(E^{*}\right)$-module.

(ii) $H\left(E^{*}\right)$ is generated by all powers of all polynomials $f$ satisfying the following defining condition:

$$
f(X)=\sum_{i, j} A_{i j} X_{i j} \quad \text { with } A A^{t}=0 ; i=1, \ldots, n ; j=1, \ldots, k .
$$

Here $A_{i j}$ are entries of a given matrix $A \in E$.

Definitions 0.7. Let $H(E, \xi)$ denote the subspace of $P(E, \xi)$ consisting of all $G$-harmonic $\xi$-covariant polynomial functions. An element $F$ of $P(E, \rho)$ will 
be called $G$-harmonic if $\partial(l \cdot F)=0$ for all linear functionals $l$ on $\operatorname{Hol}(\mathrm{GL}(n, \mathbf{C}), \xi)$, and for every homogeneous differential operator $\partial$ with constant coefficients and without constant term that commutes with $G$. Now let $H(E, \rho)$ denote the subspace of $P(E, \rho)$ consisting of all $G$-harmonic $\rho$-covariant polynomial mappings. Let $R(\cdot, \xi)$ (resp. $R_{0}(\cdot, \xi)$ ) be the representation of $G$ (resp. the representation of $G_{0}$ ) obtained by right translation on $H(E, \xi)$. Similarly we define $R(\cdot, \rho)$ and $R_{0}(\cdot, \rho)$, representations of $G$ and $G_{0}$, respectively, on $H(E, \rho)$.

THEOREM 3.1. If $k>2 n$, the representation $R_{0}(\cdot, \xi)$ of $G_{0}$ on $H(E, \xi)$ is irreducible and its highest weight is indexed by

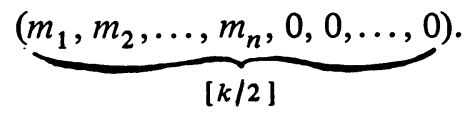

COROLLARY 3.2. If $k>2 n$, the representation $R(\cdot, \rho)$ of $G$ on $H(E, \rho)$ is equivalent to the irreducible representation $R(\cdot, \xi)$ of $G$ on $H(E, \xi)$.

DEFINITIONS 0.8. Let $S^{n, k}$ denote the Stiefel manifold consisting of all matrices $s \in \mathrm{R}^{n \times k}$ such that $s s^{t}=1_{n}, 1_{n}$ being the unit matrix of order $n$. Let $H\left(S^{n, k}\right)$ denote the space of functions on $S^{n, k}$ obtained by restricting all $G$-harmonic polynomials to the Stiefel manifold.

THEOREM 4.2. If $k>2 n$, the restriction mapping $f \rightarrow f / S^{n, k}\left(f \in H\left(E^{*}\right)\right)$ of $H\left(E^{*}\right)$ onto $H\left(S^{n, k}\right)$ is a $G_{0}$-module isomorphism.

REMARKS. Theorem 4.2 was proved by D. Levine [17] for the case $n=2$. S. Gelbart [7], D. Levine [17] , and R. Strichartz [22] investigated the $\rho$-covariant type of harmonic polynomials and Stiefel harmonic functions. Theorem 2.5 (ii) was stated in [7] as a conjecture of E. M. Stein. I am indebted to Professor K. Okamoto for his conjecture concerning Theorem 3.1. This paper is in essence my doctoral dissertation. It is a great pleasure to me to express here my profound respect, gratitude, and admiration for my adviser, Professor Ray A. Kunze.

1. The study of the representation $D(\cdot, \rho)$. Since the Borel-Weil-Bott theorem, or, equivalently, Zhelobenko's work in [27] and [28] give a complete classification of the irreducible finite dimensional representations of $\operatorname{GL}(n, \mathbf{C})$, it is natural to get a concrete realization of $P(E, \rho)$ following those theories. Since we shall use it frequently throughout our investigation, a brief summary of the Borel-WeilBott theorem seems necessary. For details and justifications see [2], [3], [16], [25], [27]. and [28]. For the terminology introduced in this section see [1].

Suppose that $G_{0}$ is a compact, connected, semisimple Lie group. Let $T$ be a maximal torus of $G_{0}$. Let $G$ be the complexification of $G_{0}$ and $B$ a Borel subgroup of $G$; then $G=B G_{0}$ and $B \backslash G=T \backslash G_{0}$. If $\xi \in \hat{T}$, then $\xi$ extends uniquely to a holomorphic homomorphism, which we shall denote by the same symbol 
$\xi: B \rightarrow C^{*}$. Let $\operatorname{Hol}(G, \xi)$ be the space of all holomorphic functions $f$ on $G$ which also satisfy $f(b g)=\xi(b) f(g)$ for all $(b, g) \in B \times G$. Then it is well known that $\operatorname{Hol}(G, \xi)$ is finite dimensional (see $[16$, p. 365] or $[3$, p. 215]). Let $\pi(\cdot, \xi)$ | be the representation of $G$ on $\operatorname{Hol}(G, \xi)$ which is given by $(\pi(g, \xi) f)(x)=f(x g)$ for all $x, g \in G$. In this context we have

THEOREM 1.1 (BOREL-WEIL-BOTT).

(1) The space $\operatorname{Hol}(G, \xi)$ is nonzero if and only if $\xi$ is a dominant weight.

(2) The representation $\pi(\cdot, \xi)$ is irreducible if $\xi$ is dominant. In this case, if $\pi(\cdot, \xi) / G_{0}$ denotes the restriction of $\pi(\cdot, \xi)$ to $G_{0}$, then $\pi(\cdot, \xi) / G_{0}$ is irreducible, and its highest weight is $\xi$.

For a proof of this theorem see [3]; for the notion of dominant weight see [2] and [16, (351)].

REMARK 1.2. Theorem 1.1 shows that one can obtain every irreducible representation of a compact, connected, semisimple Lie group by the above inducing process.

In [27] and [28] Zhelobenko obtained an equivalent version of the BorelWeil-Bott theorem which we shall use when convenient.

Now let $U(n)$ denote the group of unitary matrices of order $n$. Let $\xi=$ $\xi\left(m_{1}, m_{2}, \ldots, m_{n}\right)$ be a character defined on the diagonal subgroup $T$ of $U(n)$ by $\xi(d)=d_{11}^{m_{1}} d_{22}^{m_{2}} \cdots d_{n n}^{m_{n}}$ where $d \in T$ and $m_{1} \geqslant m_{2} \geqslant \cdots \geqslant m_{n}$. Let $B$ be the lower triangular subgroup of $\mathrm{GL}(n, \mathrm{C})$ and define an extension of $\xi$ to $B$ (which we shall denote again by $\xi$ ) by setting $\xi(b)=b_{11}^{m_{1}} b_{22}^{m_{2}} \cdots b_{n n}^{m_{n}}$ for all $b \in B$. Let $\operatorname{Hol}(\mathrm{GL}(n, \mathbf{C}), \xi)$ denote the space of all $\xi$-covariant holomorphic functions on $\mathrm{GL}(n, \mathrm{C})$. The representation $\pi(\cdot, \xi)$ of $\mathrm{GL}(n, \mathrm{C})$ obtained by right translation on $\operatorname{Hol}(\mathrm{GL}(n, \mathrm{C}), \xi)$ will be denoted by $\rho$. Define $P(E, \rho)$ as the linear space of all $\rho$-covariant polynomial mappings from $E$ into $\operatorname{Hol}(\mathrm{GL}(n, \mathrm{C}), \xi)$. Let $D(\cdot, \rho)$ be the representation of $\operatorname{GL}(k, \mathrm{C})$ on $P(E, \rho)$ obtained by right translation.

THEOREM 1.3. (i) $P(E, \rho)$ is nonzero if and only if $m_{1} \geqslant m_{2} \geqslant \cdots \geqslant m_{n}$ $\geqslant 0$.

(ii) If $P(E, \rho)$ is nonzero, then the representation $D(\cdot, \rho)$ is irreducible and its highest weight is indexed by

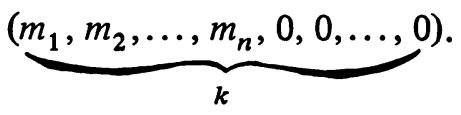

Proof. Let $B_{k}$ be the Borel subgroup of $\mathrm{GL}(k, \mathrm{C})$ consisting of all nonsingular lower triangular matrices of order $k$. $B$ can be embedded in $B_{k}$ by the injection $b \subset\left[\begin{array}{ll}b & 0 \\ 0 & 1\end{array}\right]$ for all $b \in B, 1$ being the identity matrix of order $k-n$. The character $\xi=\xi\left(m_{1}, m_{2}, \ldots, m_{n}\right)$ "extends" to a character $\widetilde{\xi}=$ 
$\widetilde{\xi}\left(m_{1}, m_{2}, \ldots, m_{n}, 0, \ldots, 0\right)$ of $B_{k}$. Define $P(\operatorname{GL}(k, \mathbf{C}), \widetilde{\xi})$ as the space of all polynomial functions $h$ on $\mathrm{GL}(k, \mathrm{C})$ which also satisfy $h\left(b_{k} y\right)=\widetilde{\xi}\left(b_{k}\right) h(y)$ for all $b_{k} \in B_{k}, y \in \mathrm{GL}(k, \mathrm{C})$ (by polynomial functions on $\mathrm{GL}(k, \mathrm{C})$ we mean restriction to $\mathrm{GL}(k, \mathrm{C})$ of polynomial functions on $\left.\mathrm{C}^{k \times k}\right)$. Let $\pi(\cdot, \widetilde{\xi})$ be the representation of $\mathrm{GL}(k, \mathrm{C})$ on $P(\mathrm{GL}(k, \mathrm{C}), \widetilde{\xi})$ obtained by right translation. By Theorem 1.1, we know that $\pi(\cdot, \widetilde{\xi})$ is irreducible with highest weight $\widetilde{\xi}\left(m_{1}, m_{2}, \ldots, m_{n}, 0, \ldots, 0\right)$. Now for a fixed $y \in \mathrm{GL}(k, \mathrm{C})$ the mapping $b_{k} \rightarrow b_{k} y\left(b_{k} \in B_{k}\right)$ is polynomial. If $h$ $\in P(\mathrm{GL}(k, \mathrm{C}), \widetilde{\xi})$ then the mapping $b_{k} \rightarrow h\left(b_{k} y\right)$ is polynomial. This fact together with the equality $h\left(b_{k} y\right)=\widetilde{\xi}\left(b_{k}\right) h(y)$ implies that $P(\mathrm{GL}(k, \mathrm{C}), \widetilde{\xi})$ is nonzero if and only if $m_{1} \geqslant m_{2} \geqslant \cdots \geqslant m_{n} \geqslant 0$.

Note 1.4. If we represent $b_{k} \in B_{k}$ in block form as $b_{k}=\left[\begin{array}{ll}b & 0 \\ c & d\end{array}\right]$, where $b$ is an $n$ by $n$ matrix, then $b \in \underset{\sim}{ } B$ and clearly $\widetilde{\xi}\left(b_{k}\right)=\xi(b)$. Let $h_{\widetilde{\xi}}$ be the highest weight vector of $P(\mathrm{GL}(k, \mathrm{C}), \widetilde{\xi})$; then $h_{\widetilde{\xi}}(y)=\prod_{i=1}^{n}\left[D_{i}(y)\right]^{m_{i}-m_{i+1}}$ where $D_{i}(y)$ is the principal minor of order $i$ of the matrix $y, i=1, \ldots, n$ and $m_{n+1}=0$, so that as a function of $y \in \mathrm{GL}(k, \mathrm{C}), h_{\widetilde{\xi}}$ depends only on the first $n$ rows of the $k$ by $k$ matrix $y$ (cf. $[28, \S 4$, pp. 20-26] for details). Since $P(\mathrm{GL}(k, \mathrm{C}), \widetilde{\xi})$ is cyclically spanned by $h_{\widetilde{\xi}}$, we observe that the same conclusion holds for all $h \in$ $P(\mathrm{GL}(k, \mathrm{C}), \widetilde{\xi})$. More precisely, if we represent a matrix $y \in \mathrm{GL}(k, \mathrm{C})$ in block form as $y=\left[\begin{array}{l}X(y) \\ Z\end{array}\right]$ where $X(y)$ is an $n$ by $k$ matrix and $Z(y)$ is a $k-n$ by $k$ matrix, then for $h \in P(\mathrm{GL}(k, \mathrm{C}), \widetilde{\xi}), h(y)$ is independent of $Z(y)$. Since $h$ is polynomial, we can actually consider that the domain of definition of $h$ is $\mathrm{C}^{n \times k}=E$.

Now let $P(E, \xi)$ be the linear space consisting of all polynomial functions defined on $E$ which also satisfy $f(b X)=\xi(b) f(X)$ for all $(b, X) \in B \times E$. Define $D(\cdot, \xi)$ as the representation of $\mathrm{GL}(k, \mathrm{C})$ on $P(E, \xi)$ obtained by right translation. We shall prove that $D(\cdot, \xi)$ and $\pi(\cdot, \widetilde{\xi})$ are equivalent; hence $D(\cdot, \xi)$ is irreducible with highest weight $\widetilde{\xi}\left(m_{1}, m_{2}, \ldots, m_{n}, 0, \ldots, 0\right)$. For this purpose, we define a mapping $\Psi$ from $P(\mathrm{GL}(k, \mathrm{C}), \widetilde{\xi})$ to $P(E, \xi)$ in the following fashion: For every $X \in E$, we represent $X$ in block form as $[u(X) v(X)]$ where $u(X)$ (resp. $v(X)$ ) is an $n$ by $n$ (resp. $n$ by $k-n$ ) matrix; let $y[X]=\left[\begin{array}{c}u(X) \\ 0\end{array}{ }_{1}(X)\right], 1$ being the unit matrix of order $k-n$. Now for all $h \in P(\mathrm{GL}(k, \mathrm{C}), \widetilde{\xi}), X \in E$, we set $(\Psi h)(X)=h(y[X])$; the above observation (Note 1.4) together with the fact that

$$
(\Psi h)(b X)=h(y[b X])=h\left(\left[\begin{array}{ll}
b & 0 \\
0 & 1
\end{array}\right] y[X]\right)=\xi(b) h(y[X])=\xi(b)(\Psi h)(X)
$$

for all $(b, X) \in B \times E$ show that $\Psi$ is well defined and injective. $\Psi$ is clearly linear. For every $f \in P(E, \xi)$ we define a polynomial function $h$ on $\mathrm{GL}(k, \mathrm{C})$ by setting $h(y)=f(X(y))$ for all $y \in \mathrm{GL}(k, \mathrm{C})$ represented in block form as $y=$ $\left[\begin{array}{l}X(y) \\ Z(y)\end{array}\right]$. Now for all $b_{k} \in B_{k}$ we have

$h\left(b_{k} y\right)=f\left(X\left(b_{k} y\right)\right)=f(b X(y))=\xi(b) f(X(y))=\widetilde{\xi}\left(b_{k}\right) h(y)$ if $b_{k}=\left[\begin{array}{ll}b & 0 \\ c & d\end{array}\right]$. 
Hence, $h \in P(\mathrm{GL}(k, \mathrm{C}), \widetilde{\xi})$. Now $(\Psi h)(X)=h(y[X])=f(X)$ for all $X \in E$, so $\Psi h=f$. This shows that $\Psi$ is surjective and $\left(\Psi^{-1} f\right)(y)=f(X(y))$ for all $f \in$ $P(E, \xi)$. For every $y_{1} \in \mathrm{GL}(k, \mathrm{C}), X \in E$ and $h \in P(\mathrm{GL}(k, \mathrm{C}), \widetilde{\xi})$ we have

$$
\begin{aligned}
\left(\Psi \pi\left(y_{1}, \widetilde{\xi}\right) h\right)(X) & =\pi\left(y_{1}, \widetilde{\xi}\right) h(y[X])=h\left(y[X] y_{1}\right) \\
& =h\left(y\left[X y_{1}\right]\right)=\Psi h\left(X y_{1}\right)=\left(D\left(y_{1}, \xi\right) \Psi h\right)(X) .
\end{aligned}
$$

Equation (1.1) shows that $\Psi$ interwines $\pi(\cdot, \tilde{\xi})$ and $D(\cdot, \xi)$

The following result, an "induction in stages," which is interesting in its own right, will be used in the proof of Theorem 1.3.

THEOREM 1.5. If $D(\cdot, \xi)($ resp. $D(\cdot, \rho))$ denotes the representation of $\mathrm{GL}(k, \mathrm{C})$ obtained by right translation on the linear space $P(E, \xi)($ resp. $P(E, \rho)$ ) of all $\xi$-covariant (resp. $\rho$-covariant) complex-valued polynomial functions on $E$ (resp. polynomial mappings from $E$ into $\operatorname{Hol}(\mathrm{GL}(n, \mathrm{C}), \xi))$, then, under the assumption $m_{1} \geqslant m_{2} \geqslant \cdots m_{n} \geqslant 0, D(\cdot, \xi)$ and $D(\cdot, \rho)$ are equivalent.

Proof. Define a map $\Phi$ from $P(E, \xi)$ to $P(E, \rho)$ by $[(\Phi f)(X)](a)=f(a X)$ for all $a \in \mathrm{GL}(n, \mathbf{C}), X \in E$, and $f \in P(E, \xi)$. Then $(\Phi f)(X) \in \operatorname{Hol}(\mathrm{GL}(n, \mathbf{C}), \xi)$, because

$$
[(\Phi f)(X)](b a)=f(b a X)=\xi(b) f(a X)=\xi(b)[(\Phi f)(X)](a)
$$

for all $a \in \mathrm{GL}(n, \mathbf{C}), b \in B$, and $X \in E$. Clearly $\Phi f$ is a polynomial mapping from $E$ into $\operatorname{Hol}(\mathrm{GL}(n, \mathrm{C}), \xi)$, and since for every $a, a_{1} \in \mathrm{GL}(n, \mathrm{C})$, and $X \in E$, we have

$$
\left[(\Phi f)\left(a_{1} X\right)\right](a)=f\left(a a_{1} X\right)=[(\Phi f)(X)]\left(a a_{1}\right)=\left[\rho\left(a_{1}\right)(\Phi f)(X)\right](a),
$$

it follows that $\Phi f$ belongs to $P(E, \rho)$. It is also clear that $\Phi$ is linear. Let us show that $\Phi$ is injective. Indeed if $(\Phi f)(X)=0$ for all $X \in E$, then $[(\Phi f)(X)](a)=0$ for all $a \in \mathrm{GL}(n, \mathrm{C})$; in particular; for $a$ equal 1 , the identity matrix of order $n$, we get $[\Phi f(X)](1)=f(1 X)=f(X)=0$ for all $X \in E$, so $f=0$.

To see that $\Phi$ is surjective we suppose that $F \in P(E, \rho)$ and define $f$ by $f(X)=(F(X))(1)$ for all $X \in E$. Clearly $f$ is a polynomial function and

$$
\begin{aligned}
f(b X) & =F(b X)(1)=[\rho(b) F(X)](1)=(F(X))(1 b) \\
& =(F(X))(b 1)=\xi(b)(F(X))(1)=\xi(b) f(X) .
\end{aligned}
$$

Therefore, $f$ belongs to $P(E, \xi)$. Now for all $(a, X) \in \mathrm{GL}(n, \mathrm{C}) \times E$ we have

$$
\begin{aligned}
{[(\Phi f)(X)](a) } & =f(a X)=(F(a X))(1)=[\rho(a) F(X)](1) \\
& =F(X)(1 a)=(F(X))(a),
\end{aligned}
$$

so that $\Phi f=F$. Therefore, $\Phi$ is an isomorphism and its inverse mapping is given by $\left(\Phi^{-1} F\right)(X)=(F(X))(1)$.

For all $y \in \mathrm{GL}(k, \mathbf{C}), a \in \mathrm{GL}(n, \mathbf{C}), X \in E$, and $f \in P(E, \xi)$ we have 


$$
\begin{aligned}
{[(D(y, \rho) \Phi f)(X)](a) } & =[(\Phi f)(X y)](a)=f(a X y) \\
& =(D(y, \xi) f)(a X)=[\Phi D(y, \xi) f(X)](a) .
\end{aligned}
$$

Equation (1.2) shows that $\Phi$ intertwines $D(\cdot, \rho)$ and $D(\cdot, \xi)$. This achieves the proof of Theorem 1.5 and also completes that of Theorem 1.3.

REMARK 1.6. The proof of Theorem 1.3 (via Theorem 1.5) shows that the space of all complex-valued $\xi$-covariant polynomial functions $P(E, \xi)$ is naturally isomorphic to the space of all vector-valued $\rho$-covariant polynomial mappings $P(E, \rho)$. In fact when $n=1$ and $k>2, \xi=\xi\left(m_{1}\right), m_{1} \geqslant 0$, so that $P(E, \xi)$ is the space of all homogeneous polynomials in $k$ variables of degree $m_{1}$. Hence, the notion of $\xi$-covariance as well as that of $\rho$-covariance are equivalent generalizations of the notion of homogeneity. Also the study of the restriction of $D(\cdot, \rho)$ to $G$ or $G_{0}$ is equivalent to that of the restriction of $D(\cdot, \xi)$ to $G$ or $G_{0}$. This naturally reduces our problems to the investigation of the symmetric algebra $S\left(E^{*}\right)$ of polynomial functions on $E$. In this context, there is a close connection between our investigation and the work of S. Helgason [12], B. Kostant [15], and $H$. Maass [18], [19]. In the next section, we shall consider this connection.

2. The "separation of variables" theorem for the symmetric algebra $S\left(E^{*}\right)$. In this section we shall study the algebra of all complex-valued polynomial functions on $\mathbf{C}^{n \times k}$ under the assumption $k>2 n$. The rings of differential operators and polynomial functions on a finite dimensional vector space, invariant under linear actions of certain semisimple Lie groups, were investigated abstractly and in great detail by Harish-Chandra [10], S. Helgason [12], and B. Kostant [15]. We shall begin by reformulating some of these notions in a manner convenient to our particular problem.

The most important results of this section are: $1^{\circ}$ the "separation of variables" theorem; $2^{\circ}$ Corollary 2.7 ; the latter will play an important role in the proof of Theorem 3.1 of $\S 3$.

Recall that $E_{0}, E, G_{0}$, and $G$ stand for $\mathbf{R}^{n \times k}, \mathbf{C}^{n \times k}, S O(k)$, and $S O(k, \mathbf{C})$, respectively. Let $E^{*}$ denote the dual of $E$; and let $S\left(E^{*}\right)$ be the algebra of all complex-valued polynomial functions on $E$. Thus $S\left(E^{*}\right)$ is generated by $E^{*}$ and the constant functions. For $f$ in $S\left(E^{*}\right)$ let $D_{i j} f$ be the partial derivative of $f$ with respect to the $i j$ coordinate, $1 \leqslant i \leqslant n ; 1 \leqslant j \leqslant k$. If $p$ is any element of $S\left(E^{*}\right)$ let $p(D)$ denote the differential operator obtained from $p(Y)$ by replacing $Y_{i j}$ with $D_{i j}$. For $X$ in $E$ let $X^{*}(Y)=\operatorname{tr}\left(X Y^{t}\right)$, where tr denotes the trace function and $Y^{t}$ the transpose of $Y$. Clearly $X^{*}$ belongs to $E^{*}$. Now we define a linear action of $\mathrm{GL}(k, \mathrm{C})$ on $S\left(E^{*}\right)$ by setting $(R(y) p)(X)=p(X y)$ for all $y \in \mathrm{GL}(k, \mathrm{C})$, $X \in E$, and $p \in S\left(E^{*}\right)$. This action, when restricted to $G$, induces a structure of $G$-module on $S\left(E^{*}\right)$. For $y \in \mathrm{GL}(k, \mathrm{C}), R(y) X^{*}=\left(X y^{t}\right)^{*}$. For each $X$ in $E$, 


$$
\left(X^{*}(D) f\right)(Y)=\sum_{i j} X_{i j}\left(D_{i j} f\right)(Y)=\{(d / d t) f(Y+t X)\}_{t=0}
$$

Hence,

Therefore,

$$
\begin{aligned}
\left(R(y) X^{*}(D) R\left(y^{-1}\right) f\right)(Y) & =\left(X^{*}(D) R\left(y^{-1}\right) f\right)\left(Y_{y}\right) \\
& =\left\{(d / d t)\left(R\left(y^{-1}\right) f\right)(Y y+t X)\right\}_{t=0} \\
& =\left\{(d / d t) f\left(Y+t X y^{-1}\right)\right\}_{t=0}
\end{aligned}
$$

$$
R(y) X^{*}(D) R\left(y^{-1}\right)=\left(X y^{-1}\right) *(D)
$$

For any $Y$ in $E$,

$$
\begin{aligned}
\left(X y^{-1}\right)^{*}(Y) & =\operatorname{tr}\left(X y^{-1} Y^{t}\right)=\operatorname{tr}\left(X\left(Y\left(y^{-1}\right)^{t}\right)^{t}\right) \\
& =\left(R\left(y^{\sqrt{ }}\right) X^{*}\right)(Y) \quad\left(y^{\sqrt{ }}=\left(y^{-1}\right)^{t}\right)
\end{aligned}
$$

It follows that

$$
R(y) p(D) R\left(y^{-1}\right)=\left(R\left(y^{\sqrt{ }}\right) p\right)(D) \text { for all } p \text { in } S\left(E^{*}\right) .
$$

Indeed, to establish relation (2.1) we let $C$ be the class of polynomials for which (2.1) is true. Let $p, q \in C$. Then

$$
\begin{gathered}
R(y)(p+q)(D) R\left(y^{-1}\right)=R(y)(p(D)+q(D)) R\left(y^{-1}\right)=\left(R\left(y^{\sqrt{ }}\right)(p+q)\right)(D) . \\
R(y)(p q)(D) R\left(y^{-1}\right)=R(y) p(D) q(D) R\left(y^{-1}\right)=\left(R\left(y^{\sqrt{ }}\right)(p q)\right)(D) .
\end{gathered}
$$

Moreover, $C$ contains $X^{*}$ for each $X$ in $E$; hence $C=S\left(E^{*}\right)$.

For any two polynomials $p$ and $q$ in $S\left(E^{*}\right)$ let $\langle p, q\rangle=(p(D) q)(0)$. Then for $y \in O(k, \mathrm{C}),\langle R(y) p, R(y) q\rangle=\langle p, q\rangle$ by $(2.1)$ and the fact that $y^{\sqrt{ }}=y$. Notice that $\left\langle X^{*}, Y^{*}\right\rangle=\operatorname{tr}\left(X Y^{t}\right)$. Let $S^{r}\left(E^{*}\right)(r \in \mathrm{N})$ denote the subspace of $S\left(E^{*}\right)$ consisting of all homogeneous polynomials of degree $r$. If $t=n k$ let $\left\{X_{1}^{*}, X_{2}^{*}, \ldots, X_{t}^{*}\right\}$ be a basis for $E^{*}$ such that $\left\langle X_{i}^{*}, X_{j}^{*}\right\rangle=\delta_{i j}$. Then if

$$
p=\sum_{m_{i} \geqslant 0} a\left(m_{1}, \ldots, m_{t}\right) X_{1}^{* m_{1}} X_{2}^{* m_{2}} \cdots X_{t}^{* \dot{m}_{t}}
$$

and

$$
q=\sum_{m_{i} \geqslant 0} b\left(m_{1}, \ldots, m_{t}\right) X_{1}^{* m_{1}} X_{2}^{* m_{2}} \cdots X_{t}^{* m_{t}}
$$

$\left(a\left(m_{1}, \ldots, m_{t}\right), b\left(m_{1}, \ldots, m_{t}\right)\right.$ are complex numbers and $p, q$ belong to $\left.S^{r}\left(E^{*}\right)\right)$, we have

$$
\langle p, q\rangle=\sum m_{1} ! \cdots ! m_{t} ! a\left(m_{1}, \ldots, m_{t}\right) b\left(m_{1}, \ldots, m_{t}\right) .
$$

It follows immediately from $(2.2)$ that $\langle\cdot, \cdot\rangle$ is a symmetric nondegenerate bilinear form on $S\left(E^{*}\right) \times S\left(E^{*}\right)$. Moreover, if $p$ and $q$ are homogeneous polynomials of different degrees, $\langle p, q\rangle=0$. 
Definitions 2.1. A polynomial $p \in S\left(E^{*}\right)$ is said to be $G$-invariant if $R(g) p=p$ for all $g \in G$. A differential operator $p(D)$ is said to be $G$-invariant if it commutes with the action of $G$ on $S\left(E^{*}\right)$; i.e., $R(g) p(D)=p(D) R(g)$ for all $g$ in $G$.

From the fact that $\langle\cdot, \cdot\rangle$ is nondegenerate, we infer that the map $p \rightarrow$ $p(D)$ is an algebra isomorphism. This, together with relation $(2.1)$, shows that $p$ is $G$-invariant if and only if $p(D)$ is $G$-invariant; i.e.,

$R(g) p(D) R\left(g^{-1}\right)=p(D)$ for all $g \in G \Leftrightarrow R(g) p=p$ for all $g \in G$.

Note 2.2. In Definitions 2.1 if we replace $G$ by $G_{0}$, then relation (2.3) still holds for all $g \in G_{0}$.

Now we equip $S^{r}\left(E^{*}\right)$ with an inner product defined in the following fashion: If $q \in S^{r}\left(E^{*}\right)$,

$$
q=\sum b\left(m_{1}, \ldots, m_{t}\right) X_{1}^{* m_{1}} X_{2}^{* m_{2}} \cdots X_{t}^{* m_{t}},
$$

we let

$$
\widetilde{q}=\sum \bar{b}\left(m_{1}, \ldots, m_{t}\right) X_{1}^{* m}{ }_{1} X_{2}^{* m_{2}} \cdots X_{t}^{* m_{t}} \text {, }
$$

where $\bar{b}\left(m_{1}, \ldots, m_{t}\right)$ is the complex-conjugate of $b\left(m_{1}, \ldots, m_{t}\right)$; now set

$$
(p, q)=\langle p, \widetilde{q}\rangle \text { for all } p, q \text { in } S^{r}\left(E^{*}\right) .
$$

For $n=1$ this is the same inner product as defined in [21, p. 139]. Moreover, this inner product extends obviously to an inner product on $S\left(E^{*}\right)$. It follows from the above paragraphs that $G_{0}$ leaves $(\cdot, \cdot)$ invariant; i.e. $(R(g) p, R(g) q)=$ $(p, q)$ for all $p, q$ in $S\left(E^{*}\right)$ and for all $g$ in $G_{0}$.

Now let $S^{c}\left(E_{0}^{*}\right)$ denote the algebra of all complex-valued polynomial functions on $E_{0}$. Each polynomial function $f \in S^{c}\left(E_{0}^{*}\right)$ extends uniquely to a polynomial function on $E$, also denoted by $f$. Therefore the restriction mapping $f \rightarrow$ $f / E_{0}\left(f \in S\left(E^{*}\right)\right)$ is an isomorphism between $S\left(E^{*}\right)$ and $S^{c}\left(E_{0}^{*}\right)$.

Let $J\left(E^{*}\right)$ (resp. $J^{c}\left(E_{0}^{*}\right)$ ) be the subring of $S\left(E^{*}\right)$ (resp. $S^{c}\left(E_{0}^{*}\right)$ ) consisting of all $G$-invariant (resp. $G_{0}$-invariant) polynomials. In this context we have the following proposition which can also be found in a more general form in $[9$, Lemma 1.5, p. 246].

Proposition 2.3. Under the restriction mapping $f \rightarrow f / E_{0} \quad\left(f \in S\left(E^{*}\right)\right)$ from $S\left(E^{*}\right)$ onto $S^{c}\left(E_{0}^{*}\right), J\left(E^{*}\right)$ is mapped isomorphically onto $J^{c}\left(E_{0}^{*}\right)$.

Proof. The proof is elementary. For details see [23].

Let $J_{+}\left(E^{*}\right)$ (resp. $J_{+}^{c}\left(E_{0}^{*}\right)$ ) denote the subset of $J\left(E^{*}\right)$ (resp. $J^{c}\left(E_{0}^{*}\right)$ ) consisting of all $G$-invariant (resp. $G_{0}$-invariant) polynomial functions without constant term. It is well known, from the theory of polynomial invariants (cf. [26, Chapter II] ), that $J_{+}^{c}\left(E_{0}^{*}\right)$ is the linear space generated by all products of the polynomials $p_{i j}$, 
$1 \leqslant i \leqslant j \leqslant n$, defined by

$$
p_{i j}(X)=\sum_{s=1}^{k} X_{i s} X_{j s}, \quad \text { where } X=\left(X_{r s}\right) \in E_{0}, 1 \leqslant r \leqslant n, 1 \leqslant s \leqslant k .
$$

From Proposition 2.3 we infer that $J_{+}\left(E^{*}\right)$ is generated by the same polynomials $p_{i j}, 1 \leqslant i \leqslant j \leqslant n$, as defined by (2.5) but now their domain of definition is $E$.

Definition 2.4. A polynomial function $f \in S\left(E^{*}\right)$ (resp. $f \in S^{c}\left(E_{0}^{*}\right)$ ) will be called $G$-harmonic (resp. $G_{0}$-harmonic) if $p(D) f=0$ for all $p \in J_{+}\left(E^{*}\right)$ (resp. $\left.p \in J_{+}^{c}\left(E_{0}^{*}\right)\right)$.

Let $H\left(E^{*}\right)$ denote the subspace of $S\left(E^{*}\right)$ consisting of all $G$-harmonic polynomial functions. Then from the above paragraphs we deduce that

$$
H\left(E^{*}\right)=\left\{f \in S\left(E^{*}\right): \Delta_{i j} f=0,1 \leqslant i \leqslant j \leqslant n ; \text { where } \Delta_{i j} f=p_{i j}(D) f\right\} .
$$

Notice that when $n=1, J_{+}\left(E^{*}\right)$ is spanned by all powers of a single polynomial $p(X)=p_{11}(X)=\Sigma_{i=1}^{k} X_{i}^{2}$. Hence, $H\left(E^{*}\right)$ is then reduced to the space of all "classical" harmonic polynomial functions in $k$ variables.

Let $P$ denote the algebraic variety in $E$ defined by

$$
P=\left\{X \in E: f(X)=0 \text { for all } f \in J_{+}\left(E^{*}\right)\right\} .
$$

From relation (2.5) we see immediately that

$$
P=\left\{X \in E: X X^{t}=0\right\} .
$$

Set

$$
P_{n}=\{X \in P: \operatorname{rank}(X)=n\} .
$$

For the remainder of this section we shall always assume that $k>2 n$ (although occasionally we shall make some remarks about the case $k=2 n$ ). This restriction, which occurs also in the classical case when $n=1$, seems to be necessary both from the point of view of algebraic geometry as well as from the standpoint of the representation theory of our problem.

THEOREM 2.5 ("Separation of variables" theorem for $\mathbf{C}^{n \times k}, k>2 n$ ).

(i) The algebra $S\left(E^{*}\right)$ of all complex-valued polynomial functions on $E$ is spanned by all products of the form $j h$ where $j$ is $G$-invariant and $h$ is G-harmonic. In fact, $S\left(E^{*}\right)=J\left(E^{*}\right) \otimes H\left(E^{*}\right)$ and $S\left(E^{*}\right)$ is a free $J\left(E^{*}\right)$-module.

(ii) The space $H\left(E^{*}\right)$ of $G$-harmonic polynomials is generated by all powers of all polynomials $f$ satisfying the following defining condition:

$$
f(X)=\sum_{i j j} A_{i j} X_{i j} \quad \text { with } A A^{t}=0 ; i=1, \ldots, n ; j=1, \ldots, k .
$$

Here the $A_{i j}$ are entries of a given matrix $A \in E$.

In particular, the subspace $H^{r}\left(E^{*}\right)$ of all G-harmonic homogeneous polynomials 
of a fixed degree $r \geqslant 0$ is spanned by the polynomials $f^{r}$, fbeing defined by condition (2.8).

Note 2.6. (1) If we set $P^{*}=\left\{A^{*} \in S\left(E^{*}\right): A \in P\right\}$, and let $H_{P}\left(E^{*}\right)$ be the linear space spanned by all powers $\left(A^{*}\right)^{m}, m=1,2, \ldots$, then Theorem 2.5 (ii) simply states that $H\left(E^{*}\right)=H_{P}\left(E^{*}\right)$.

(2) In the present context our Theorem 2.5 corresponds to Theorems 1.2 and 1.3 (see also remark on p. 247) in [12] (with $H_{1}\left(E^{*}\right)=H_{P}\left(E^{*}\right)$ and $H_{2}\left(E^{*}\right)=\{0\}$ ), and to the "general development theorem" (Theorem 3) in [18] (if we consider an element $X \in \dot{E}$ as a row vector with $n k$ components. Also Theorem 3 of [18] was proved for $S^{c}\left(E_{0}^{*}\right)$, but we can see that the result is still valid for our case from the observations made in the first part of this section).

(3) In the proof of Theorem 2.5 we shall use Propositions 4 and 7 in [15] and related matters.

(4) Recall that $\xi(b)=b_{11}^{m_{1}} b_{22}^{m_{2}} \cdots b_{n n}^{m_{n}}$ for all $b \in B$. Now let $m=\sum_{i=1}^{n} m_{i}$ and $b^{\prime} \in B$ such that $b_{i i}^{\prime}=\lambda, i=1, \ldots, n, \lambda \in \mathrm{C}^{*}$; then $f \in P(E, \xi)$ implies that $f\left(b^{\prime} X\right)=$ $\lambda^{m} f(X)$. This shows immediately that $P(E, \xi)$ is a subspace of $S^{m}\left(E^{*}\right)$, the space of all homogeneous polynomial functions of degree $m$ on $E$.

Now let $S\left(P_{n}\right)$ be the ring of all functions on $P_{n}$ obtained by restricting elements of $S\left(E^{*}\right)$ to $P_{n}$. Then we have

COROLlaRy 2.7. The restriction mapping $f \rightarrow f / P_{n}\left(f \in H\left(E^{*}\right)\right)$ is a $G$ module isomorphism of $H\left(E^{*}\right)$ onto $S\left(P_{n}\right)$.

Proof of Theorem 2.5. Recall that $P_{n}=\{X \in P: \operatorname{rank}(X)=n\}$; thus $P_{n}$ is a dense submanifold of $P$. Suppose $X_{0} \in P_{n}$ is given in the block form $X_{0}$ $=\left[\left(I_{n}\right)\left(-I_{n}^{\prime}\right)(0)\right]$, where $I_{n}$ is the unit matrix of order $n, I_{n}^{\prime}=(\sqrt{-1}) I_{n}, \sqrt{-1}$ being a fixed square root of -1 .

Lemma 2.8. The set $P_{n}$ is the orbit of $X_{0}$ under the action of G, i.e. $P_{n}=$ $\mathrm{O}_{X_{0}}=X_{0} G$.

PRoof. Since $X_{0} X_{0}^{t}=0, \operatorname{rank}\left(X_{0}\right)=n$, and $g g^{t}=1_{k}$, it follows that $X_{0} g$ has rank $n$ and $\left(X_{0} g\right)\left(X_{0} g\right)^{t}=X_{0} g g^{t} X_{0}^{t}=X_{0} X_{0}^{t}=0$ for all $g \in G$. Therefore, $X_{0} G \subset P_{n}$. We shall prove that $P_{n} \subset X_{0} G$. Now let $(\cdot \mid \cdot)$ be the complex symmetric bilinear form on $\mathbf{C}^{1 \times k}$ which is defined by the equation $(x \mid y)=x y^{t}$; then it is clear that $(\cdot \mid \cdot)$ is nondegenerate. For $X \in P_{n}$ we let $V=V_{X}$ be the subspace (of $\mathbf{C}^{1 \times k}$ ) spanned by the rows of $X$. In general, we shall denote by $\left\langle x_{1}, x_{2}, \ldots, x_{j}, \ldots, x_{s}\right\rangle$ the subspace spanned by any $s$ elements $x_{j}, j=1, \ldots, s$, of $\mathrm{C}^{1 \times k}$. Since $\operatorname{rank}(X)=n, V$ is of dimension $n$; also since $X \in P_{n}, V$ is totally isotropic with respect to $(\cdot \mid \cdot)$ (i.e. $V \subset V^{\perp}$ where $V^{\perp}$ is the orthogonal complement of $V$ with respect to the bilinear form $(\cdot \mid \cdot)$ on $\left.\mathbf{C}^{1 \times k}\right)$. Since $(\cdot \mid \cdot)$ is nonsingular, the subspace $V^{\perp}$ is of dimension $k-n>n$ (if we use $(\cdot \mid \cdot)$ 
to identify $\mathbf{C}^{1 \times k}$ with its dual, then $V^{\perp}$ is identified with $V^{0}$, the annihilator of $V$, and the result follows from Theorem 16 on p. 101 of [14]). It is clear that $\left(V^{\perp}\right)^{\perp}=V$. Since $k-2 n>0, V^{\perp} / V$ is nonzero and has dimension $k-2 n$. Since $(\cdot \mid \cdot)$ vanishes on $V$, we can define a symmetric bilinear form on $V^{\perp} / V$ (which we shall denote by the same symbol, $(\cdot \mid \cdot))$ by setting $(y+V \mid z+V)=$ $(y \mid z)$ for $y$ and $z$ in $V^{\perp}$. Now if we denote the complement of $V$ in $V^{\perp}$ by $V^{\perp}-$ $V$, then for every $y$ in $V^{\perp}-V \quad\left(=V^{\perp}-\left(V^{\perp}\right)^{\perp}\right)$ there exists $z \in V^{\perp}$ such that $(y+V \mid z+V)=(y \mid z) \neq 0$. Hence $(\cdot \mid \cdot)$ is nondegenerate on $V^{\perp} / V$. Since $V^{\perp} / V$ is a finite dimensional vector space over the complex field, it is a well-known fact (see, e.g., [14, Theorem 4, p. 370]) that there exists an orthonormal basis for $V^{\perp} / V$ with respect to $(\cdot \mid \cdot)$, i.e. there exist $\alpha_{2 n+1}, \ldots, \alpha_{k} \in V^{\perp}$ such that $\left(\alpha_{i}+V \mid \alpha_{j}+V\right)=\delta_{i j}$ for all $i, j=2 n+1, \ldots, k$. It follows immediately that $\alpha_{2 n+1}, \ldots, \alpha_{k}$ form an orthonormal system in $V^{\perp}$ with respect to $(\cdot \mid \cdot)$. Now let $W=\left\langle\alpha_{2 n+1}, \alpha_{2 n+2}, \ldots, \alpha_{k}\right\rangle$; then the vectors $x_{1}, x_{2}, \ldots, x_{n}, \alpha_{2 n+1}, \ldots$, $\alpha_{k}$ are linearly independent; hence $V^{\perp}=V \oplus W$. Indeed, if $\Sigma c_{i} x_{i}+\Sigma d_{t} \alpha_{t}=$ 0 with $c_{i}, d_{t} \in \mathrm{C}, i=1, \ldots, n$, and $t=2 n+1, \ldots, k$, then $\left(\Sigma c_{i} x_{i}+\Sigma d_{t} \alpha_{t} \mid \alpha_{s}\right)$ $=c_{s}$ for all $s=2 n+1, \ldots, k$, so $d_{t}=0$ and $\Sigma c_{i} x_{i}=0$; but the $x_{i}$ 's are linearly independent by hypothesis, so $c_{i}=0$ for all $i=1, \ldots, n$.

Now let

$$
L(k, n)=\left\langle x_{1}, x_{2}, \ldots, x_{n}, \alpha_{2 n+1}, \ldots, \alpha_{k}\right\rangle=V^{\perp}
$$

and

$$
L\left(k, n^{\sqrt{ }}\right)=\left\langle x_{1}, x_{2}, \ldots, x_{n-1}, \alpha_{2 n+1}, \ldots, \alpha_{k}\right\rangle .
$$

Then $\operatorname{dim} L\left(k, n^{\sqrt{ }}\right)=k-(n+1)<k-n=\operatorname{dim} L(k, n)$. Therefore $\operatorname{dim} L\left(k, n^{\sqrt{ }}\right)^{\perp}=n+1>\operatorname{dim} L(k, n)^{\perp}=n$. Hence, there exists an element $y$ such that $\left(y \mid \alpha_{s}\right)=0$ for $s=2 n+1, \ldots, k,\left(y \mid x_{i}\right)=0$ for $i=1,2, \ldots, n-1$, and $\left(y \mid x_{n}\right) \neq 0$. If $(y \mid y) \neq 0$ let $\alpha_{2 n}$ be the quotient of $y$ by a fixed square root of $(y \mid y)$. If $(y \mid y)=0$ let $\alpha_{2 n}$ be the quotient of $y+x_{n}$ by a fixed square root of $\left(y+x_{n} \mid y+x_{n}\right)$ (in the latter case $\left(y+x_{n} \mid y+x_{n}\right)=$ $\left.2\left(x_{n} \mid y\right) \neq 0\right)$. In any event, there exists $\alpha_{2 n}$ such that $\left\{\alpha_{2 n}, \alpha_{2 n+1}, \ldots, \alpha_{k}\right\}$ is an orthonormal system, $\left(\alpha_{2 n} \mid x_{i}\right)=0$ if $i=1, \ldots, n-1$, and $\left(\alpha_{2 n} \mid x_{n}\right) \neq 0$. Now $\alpha_{2 n}$ cannot belong to $L(k, n)$. For if

$$
\alpha_{2 n}=\sum_{i=1}^{n} c_{i} x_{i}+\sum_{t=2 n+1}^{k} d_{t} a_{t}, \quad c_{i}, d_{t} \in \mathbf{C},
$$

then we see immediately that $\left(\alpha_{2 n} \mid x_{n}\right)=0$, a contradiction. Therefore, $x_{1}$, $\ldots, x_{n}, \alpha_{2 n}, \alpha_{2 n+1}, \ldots, \alpha_{k}$ are linearly independent.

Now let us proceed inductively. Suppose that for $1 \leqslant t<n$ there exist $t$ vectors $\alpha_{2 n-j+1}, 1 \leqslant j \leqslant t$, with the following properties. The system $\left\{x_{1}, x_{2}\right.$, $\left.\ldots, x_{n}, \alpha_{2 n-t+1}, \alpha_{2 n-t+2}, \ldots, \alpha_{2 n}, \alpha_{2 n+1}, \ldots, \alpha_{k}\right\}$ is linearly independent; 
the $\alpha_{s}$ 's form an orthonormal system for $2 n-t+1 \leqslant s \leqslant k ;\left(\alpha_{2 n+r} \mid x_{i}\right)=0$ for $1 \leqslant i \leqslant n$ and $1 \leqslant r \leqslant k-2 n ;\left(\alpha_{2 n-j+1} \mid x_{n-j+1}\right) \neq 0$, and $\left(\alpha_{2 n-j+1} \mid x_{i}\right)=0$ for $i=1, \ldots, n, i \neq n-j+1$.

Let

$$
L(k, n-t)=\left\langle x_{1}, \ldots, x_{n}, \alpha_{2 n-t+1}, \ldots, \alpha_{k}\right\rangle
$$

and

$$
L\left(k,(n-t)^{\sqrt{ }}\right)=\left\langle x_{1}, x_{2}, \ldots, x_{n-t}^{\sqrt{ }}, \ldots, x_{n}, \alpha_{2 n-t+1}, \ldots, \alpha_{k}\right\rangle ;
$$

here the symbol $x_{n-t}^{\sqrt{ }}$ means that the term $x_{n-t}$ is missing. Now $\operatorname{dim} L\left(k,(n-t)^{\sqrt{ }}\right)=k-n+t-1$, so

$$
\operatorname{dim} L\left(k,(n-t)^{\sqrt{ }}\right)^{\perp}=n-t+1>\operatorname{dim} L(k, n-t)^{\perp}=n-t .
$$

Therefore, there exists $y$ such that $\left(y \mid x_{i}\right)=0, i \neq n-t,\left(y \mid x_{n-t}\right) \neq 0,\left(\alpha_{s} \mid y\right)$ $=0$ for $2 n-t+1 \leqslant s \leqslant k$. If $(y \mid y) \neq 0$ let $\alpha_{2 n-t}$ be the quotient of $y$ by a fixed square root of $(y \mid y)$; if $(y \mid y)=0$ then $\left(y+x_{n-t} \mid y+x_{n-t}\right)=2\left(y \mid x_{n-t}\right)$ $\neq 0$ and $\left(y+x_{n-t} \mid x_{n-t}\right)=\left(y \mid x_{n-t}\right) \neq 0$. In this case, we let $\alpha_{2 n-t}$ be the quotient of $y+x_{n-t}$ by a fixed square root of $\left(y+x_{n-t} \mid y+x_{n-t}\right)$. In any event, there exists $\alpha_{2 n-t}$ such that $\left\{\alpha_{2 n-t}, \alpha_{2 n-t+1}, \ldots, \alpha_{k}\right\}$ is an orthonormal system and $\left(\alpha_{2 n-t} \mid x_{i}\right)=0$ if and only if $i \neq n-t$. Now the system $\left\{x_{1}, x_{2}\right.$, $\left.\ldots, x_{n}, \alpha_{2 n-t}, \ldots, \alpha_{k}\right\}$ is linearly independent. For if

$$
\alpha_{2 n-t}=\sum_{i=1}^{n} c_{i} x_{i}+\sum_{j=2 n-t+1}^{k} d_{j} \alpha_{j},
$$

then $\left(\alpha_{2 n-t} \mid x_{n-t}\right)=0$, a contradiction. So we can iterate this process until we get a basis for $\mathbf{C}^{1 \times k}$ consisting of $x_{1}, \ldots, x_{n}, \alpha_{n+1}, \alpha_{n+2}, \ldots, \alpha_{k}$ such that the $\alpha_{j}$ 's, $n+1 \leqslant j \leqslant k$, form an orthonormal system, $\left(x_{i} \mid \alpha_{2 n+r}\right)=0$ for $1 \leqslant r$ $\leqslant k-2 n, 1 \leqslant i \leqslant n$, and $\left(x_{i} \mid \alpha_{n+j}\right)=0$ if and only if $i \neq j, 1 \leqslant i, j \leqslant n$.

Now define $n$ linear functionals $f_{n+i}$ on $V$ by $f_{n+i}(x)=\left(x \mid \alpha_{n+i}\right), 1 \leqslant i$ $\leqslant n$. Then the $f_{n+i}, 1 \leqslant i \leqslant n$, form a basis for $V^{*}$, the dual of $V$. Indeed, if $\Sigma c_{i} f_{n+i}=0,1 \leqslant i \leqslant n$, then $0=\Sigma c_{i} f_{n+i}\left(x_{j}\right)=c_{j}\left(x_{j} \mid \alpha_{n+j}\right)$, but $\left(x_{j} \mid \alpha_{n+j}\right) \neq$ 0 , so $c_{j}=0$ for all $j=1, \ldots, n$. Hence, $f_{n+1}, \ldots, f_{2 n}$ is the dual of a basis $y_{1}, \ldots, y_{n}$ for $V$. Thus

$$
f_{n+j}\left(y_{i}\right)=\left(y_{i} \mid \alpha_{n+j}\right)=\delta_{i j}, \quad 1 \leqslant i, j \leqslant n .
$$

Now let $\alpha_{j}=-\sqrt{-1} y_{j}+\sqrt{-1} \alpha_{n+j}$ for all $j=1, \ldots, n$. Then $\alpha_{j}-\sqrt{-1} \alpha_{n+j}$ $=-\sqrt{-1} y_{j}$ belongs to $V \subset V^{\perp}$; so $\left(\alpha_{j}-\sqrt{-1} \alpha_{n+j} \mid x_{i}\right)=0$ for all $i, j, 1 \leqslant i$, $j \leqslant n$. This last equality is equivalent to $\left(\alpha_{j} \mid x_{i}\right)=\sqrt{-1}\left(\alpha_{n+j} \mid x_{i}\right), 1 \leqslant i, j \leqslant n$. Also, for all $j, t$ with $1 \leqslant j, t \leqslant n$ we have 


$$
\begin{aligned}
\left(\alpha_{j} \mid \alpha_{t}\right) & =\left(-\sqrt{-1} y_{j}+\sqrt{-1} \alpha_{n+j} \mid-\sqrt{-1} y_{t}+\sqrt{-1} \alpha_{n+t}\right) \\
& =\delta_{j t}+\delta_{j t}-\delta_{j t}=\delta_{j t} .
\end{aligned}
$$

Now for all $i$ with $1 \leqslant i \leqslant n$ we have

Finally

$$
\begin{aligned}
\left(\alpha_{j} \mid \alpha_{n+i}\right) & =\left(-\sqrt{-1} y_{j}+\sqrt{-1} a_{n+j} \mid \alpha_{n+i}\right) \\
& =-\sqrt{-1} \delta_{j i}+\sqrt{-1} \delta_{j i}=0
\end{aligned}
$$

$$
\begin{aligned}
\left(\alpha_{j} \mid \alpha_{2 n+r}\right)= & \left(-\sqrt{-1} y_{j}+\sqrt{-1} \alpha_{n+j} \mid \alpha_{2 n+r}\right) \\
= & 0 \text { for all } r=1, \ldots, k-2 n .
\end{aligned}
$$

In conclusion, from (2.9), (2.10), (2.11), and (2.12) we have found a basis $\left\{\alpha_{1}, \ldots, \alpha_{k}\right\}$ for $\mathbf{C}^{1 \times k}$ orthonormal with respect to the bilinear form $(\cdot, \cdot)$ and such that

$$
\begin{array}{ll}
\alpha_{2 n+1}, \ldots, \alpha_{k} \in V^{\perp} \\
\left(x_{i} \mid \alpha_{n+i}\right)=-\sqrt{-1}\left(x_{i} \mid \alpha_{i}\right) \neq 0 & \text { for } 1 \leqslant i \leqslant n, \\
\left(x_{i} \mid \alpha_{n+j}\right)=-\sqrt{-1}\left(x_{i} \mid \alpha_{j}\right)=0 & \text { if } i \neq j ; 1 \leqslant i, j \leqslant n .
\end{array}
$$

Let $g_{1}$ be the $k$ by $k$ matrix with columns $\alpha_{1}^{t}, \alpha_{2}^{t}, \ldots, \alpha_{k}^{t}$. Then $g_{1}$ belongs to the complex orthogonal group $O(k, \mathrm{C})$. By (2.13), $X g_{1}$ is an $n \times k$ matrix represented in block form as $\left[(D)\left(-D^{\prime}\right)(0)\right]$, where $D$ is an invertible diagonal matrix of order $n, D^{\prime}=\sqrt{-1} D$, and 0 is the $n$ by $k-2 n$ matrix with entries all equal to zero. Since $k>2 n$, by replacing, if necessary, $\alpha_{2 n+1}^{t}$ by $-\alpha_{2 n+1}^{t}$ we can assume that $g_{1} \in G$ and still have $X g_{1}=\left[(D)\left(-D^{\prime}\right)(0)\right]$.

Next we want to show that for every $X_{1} \in P_{n}$ of the form $\left[(D)\left(-D^{\prime}\right)(0)\right]$ as above, with

$$
D=\left[\begin{array}{llllll}
d_{1} & & & & \\
& d_{2} & & & \\
& & \cdot & & \\
& & & \cdot & \\
& & & & d_{n}
\end{array}\right], \quad d_{i} \neq 0 \text { for } 1 \leqslant i \leqslant n
$$

there exists $g_{2} \in G$ such that $X_{1} g_{2}=X_{0}$. For this purpose, we let

$$
g_{2}=\left[\begin{array}{rcc}
A & B & 0 \\
-B & A & 0 \\
0 & 0 & I_{(k-2 n)}
\end{array}\right]
$$

where $A$ (resp. $B$ ) is the $n$ by $n$ matrix with the $i$ th diagonal entry equal to $1 / 2\left(d_{i}+1 / d_{i}\right)\left(\right.$ resp. $\left.1 / 2 \sqrt{-1}\left(d_{i}-1 / d_{i}\right)\right), 1 \leqslant i \leqslant n$, and $I_{(k-2 n)}$ is the identity matrix of order $k-2 n$. Now 


$$
g_{2} g_{2}^{t}=\left[\begin{array}{ccc}
A^{2}+B^{2} & 0 & 0 \\
0 & A^{2}+B^{2} & 0 \\
0 & 0 & I_{(k-2 n)}
\end{array}\right]
$$

and it is easy to verify that $A^{2}+B^{2}=I_{n}$, the unit matrix of order $n$. Since $A$, $B$ are invertible commuting matrices we have $\operatorname{det} g_{2}=\operatorname{det}\left[A^{2}+B^{2}\right]=1$. Therefore, $g_{2} \in G$. By a simple computation one can see that $X_{1} g_{2}=X_{0}$. Now for every $X \in P_{n}$, we can find $g_{1}$ such that $X g_{1}$ is of the form $X_{1}$; let $g_{2}$ be such that $X_{1} g_{2}=X_{0}$. If $g=g_{1} g_{2}$, then $X g=X\left(g_{1} g_{2}\right)=X_{1} g_{2}=X_{0}$, and this completes the proof of Lemma 2.8 .

Now recall that the $1 / 2 n(n+1)$ polynomial generators $p_{i j}$ of $J_{+}\left(E^{*}\right)$ were defined by $p_{i j}(X)=\Sigma_{s=1}^{k} X_{i s} X_{j s}$ for $X=\left(X_{r s}\right) ; 1 \leqslant r \leqslant n ; 1 \leqslant s \leqslant k$. Next we have

LEMMA 2.9. If $X \in E$ and $\operatorname{rank}(X)=n$, then the Jacobian matrix

$$
\left.J\left(p_{11}, p_{12}, \ldots, p_{n n}\right)\right|_{X}=\left[D_{r s} p_{i j}(X)\right],
$$

$1 \leqslant s \leqslant k, 1 \leqslant r, i, j \leqslant n$, has rank $1 / 2 n(n+1)$.

Proof. We proceed by induction on $n$. If $n=1$, then a simple computation gives $\left.J\left(p_{11}\right)\right|_{X}=2 X ;$ since $\operatorname{rank}(X)=1$ it follows that rank $\left.J\left(p_{11}\right)\right|_{X}=1$ $=1(1+1) / 2$, and the assertion is verified. Now assume that the lemma is true for all $r \leqslant n-1$. Then, in particular, $\left.J\left(p_{11}, \ldots, p_{n-1, n-1}\right)\right|_{Y}$ has rank $1 / 2(n-1) n$ for all $Y$ of maximal rank in $\mathbf{C}^{(n-1) \times k}$. Next let $X \in E$ and $\operatorname{rank}(X)$ $=n$. Let $X_{1}$ be the $n-1$ by $k$ matrix formed by the first $n-1$ rows of $X$. Clearly $\operatorname{rank}\left(X_{1}\right)=n-1$. Since the rank of $\left.J\left(p_{11}, \ldots, p_{n n}\right)\right|_{X}$ is unchanged if we permute the $p_{i j}$, we may consider the Jacobian

$$
\left.J\left(p_{11}, \ldots, p_{n-1, n-1}, p_{1 n}, p_{2 n}^{m}, \ldots, p_{n n}\right)\right|_{X}
$$

where we group together all functions $p_{i n}$ involving $n(1 \leqslant i \leqslant n)$ in the last $n$ components. By a simple computation, we see that $\left.J\left(p_{11}, \ldots, p_{n-1, n-1}, p_{1 n}, p_{2 n}, p_{n n}\right)\right|_{X}$ can be represented in block form $\left[\begin{array}{ll}A & 0 \\ C\end{array}\right]$, where

$$
A=\left.J\left(p_{11}, p_{12}, \ldots, p_{n-1, n-1}\right)\right|_{X_{1}} \text { and } C=\left[\begin{array}{ccc}
X_{11} & \ldots & X_{1 k} \\
X_{21} & \ldots & X_{2 k} \\
\vdots & & \vdots \\
X_{n-1,1} & \ldots & X_{n-1, k} \\
2 X_{n, 1} & \ldots & 2 X_{n, k}
\end{array}\right]
$$

is $X$ except that the $n$th row of $C$ is the $n$th row of $X$ multiplied by the factor 2 . Clearly $\operatorname{rank}(C)=\operatorname{rank}(X)=n$, and by the inductive hypothesis $\operatorname{rank}(A)=$ $1 / 2(n-1) n$. Hence, 
$\left.\operatorname{rank} J\left(p_{11}, \ldots, p_{n n}\right)\right|_{X}=\operatorname{rank}(A)+\operatorname{rank}(C)=n+1 / 2 n(n-1)=1 / 2 n(n+1)$.

We are now in position to achieve the proof of Theorem 2.5. First, note that according to a theorem due to H. Weyl (cf. [26, Chapter II, p. 35, and Theorem $(2.17 \mathrm{~A}), \mathrm{p} .75])$, the polynomials $p_{i j}, 1 \leqslant i, j \leqslant n$, are algebraically independent. Also note that $G$ is a connected semisimple linear algebraic Lie group which is reductive in the Kostant sense. As an algebra, $J\left(E^{*}\right)$ is generated by the $1 / 2 n(n+1)$ algebraically independent homogeneous polynomials $p_{i j}(1 \leqslant$ $i, j \leqslant n$ ) and the constant function 1 . By Lemma 2.8 the orbit $O_{X_{0}}$ of $X_{0}$ is dense in $P$. Let $J_{+}\left(E^{*}\right) S\left(E^{*}\right)$ denote the ideal in $S\left(E^{*}\right)$ which is generated by $J_{+}\left(E^{*}\right)$. Then Lemma 2.9 allows us to apply Proposition 7 (p. 347) in [15] and, hence, to conclude that $J_{+}\left(E^{*}\right) S\left(E^{*}\right)$ is a prime ideal. Now all hypotheses of Proposition 4 (p. 341) in [15] are met, and therefore Theorem 2.5 follows immediately from this proposition. The conclusion for the particular subspace $H^{r}\left(E^{*}\right)$ follows from the fact that $H\left(E^{*}\right)$ is obviously graded.

Proof of Corollary 2.7. Following B. Kostant (cf. [15, p. 342]) we shall call an element $X \in E$ quasi-regular if $P \in \overline{U_{c \in \mathrm{C}^{*}} \mathrm{O}_{c X^{*}}}$. Then by Lemma 2.8 it is obvious that $X_{0}$ is quasi-regular and $S\left(O_{X_{0}}\right)=S\left(P_{n}\right)$. Proposition 5 (p. 343) of [15] is now applicable and Corollary 2.7 follows immediately from this proposition.

Remarks 2.10. (1) In the case $k=2 n$, I can prove that $P_{n}$ has exactly two orbits, and in the case $n \leqslant k<2 n$, the study of some particular cases shows that $P_{n}$ has infinitely many orbits. In the case $k=2 n$, I suspect that $J_{+}\left(E^{*}\right) S\left(E^{*}\right)$ is a radical ideal so that Theorem 2.5 is still valid. (Cf. [12, remark on p. 247] and [18, Theorem 3, p. 143].) Notice that for $n=1, k=2$, this is true.

(2) With the knowledge that $J_{+}\left(E^{*}\right) S\left(E^{*}\right)$ is a prime ideal, we could apply Theorem 3 (p. 143) and Lemma 1 (p. 145) of H. Maass' results in [18] to achieve the proof of Theorem 2.5 (see observations made in the preliminary part of this present section).

After this excursion which has led us to investigate some interesting questions in algebraic geometry, we shall now return to our main theme. Indeed, in general, by restricting $D(\cdot, \rho)$ (or equivalently $D(\cdot, \xi)$ ) to $G$, the representations obtained are not irreducible anymore. In the particular case when $n=1$ and $k>2$, the harmonic homogeneous polynomials of a fixed degree constitute an irreducible subspace of $H\left(E^{*}\right)$ under the actions of $G$ and $G_{0}$ respectively. It appears that a generalization can be made from this interesting result. This generalization is applicable not to the $G$-harmonic subspaces $H^{r}\left(E^{*}\right)$ but to the $G$ harmonic $\xi$-covariant polynomials on $E(\xi \in \hat{T})$. In the next section, we shall confine our investigation to this generalization. 
3. The $\xi$ and $\rho$-covariant harmonic polynomial functions on $E$. Let $P(E, \xi)$ and $P(E, \rho)$ be defined as in $\S 1$; we shall denote by $H(E, \xi)$ the subspace of $P(E, \xi)$ consisting of all $G$-harmonic $\xi$-covariant polynomial functions on $E$. An element $F \in P(E, \rho)$ will be called $G$-harmonic if $\Delta_{i j}(l \circ F)=0$ for all linear functionals $l$ on $\operatorname{Hol}(\mathrm{GL}(n, \mathrm{C}), \xi)$ and for all $i, j, 1 \leqslant i \leqslant j \leqslant n$ (see (2.6) for the definition of $\left.\Delta_{i j}\right)$. It is clear that the $G$-harmonic $\rho$-covariant polynomial maps constitute a subspace of $P(E, \rho)$; we shall denote it by $H(E, \rho)$. Now for $a \in$ $\mathrm{GL}(n, \mathrm{C})$ we define a linear functional $l_{a}$ on $\operatorname{Hol}(\mathrm{GL}(n, \mathrm{C}), \xi)$ by setting $l_{a}(\varphi)=$ $\varphi(a)$ for all $\varphi \in \operatorname{Hol}(\mathrm{GL}(n, \mathrm{C}), \xi)$. Since the annihilator of the set $\left\{l_{a}: a \in\right.$ $\mathrm{GL}(n, \mathrm{C})\}$ consists only of the element 0 , this set generates the dual of $\operatorname{Hol}(\mathrm{GL}(n, \mathrm{C}), \xi)$. Thus, to verify that $F \in P(E, \rho)$ is $G$-harmonic, it is sufficient to show that $\Delta_{i j}\left(l_{a} \circ F\right)=0$ for all $a \in \mathrm{GL}(n, \mathrm{C})$ and for all $\Delta_{i j}, 1 \leqslant i \leqslant j \leqslant n$.

We define an action of $B$, the lower triangular subgroup of $\mathrm{GL}(n, \mathbf{C})$, on $P(E, \xi)$ by $(L(b) f)(X)=f\left(b^{-1} X\right)$ for all $(b, X) \in B \times E$ and all $f \in P(E, \xi)$. Clearly the actions of $G$ and $B$ on $P(E, \xi)$ commute. Now by definition, for all $i, j(1 \leqslant i \leqslant j \leqslant n) \Delta_{i j}$ commutes with the action of $G$ on $S\left(E^{*}\right)$. These facts imply that $H(E, \xi)$ is a subspace of $S\left(E^{*}\right)$ invariant under both actions of $B$ and $G$ respectively. We define $R(\cdot, \xi)$ as the representation of $G$ on $H(E, \xi)$ obtained by right translation.

For every $a \in \mathrm{GL}(n, \mathrm{C})$ and every $F \in P(E, \rho)$ we notice that $l_{a} \circ F \in$ $S\left(E^{*}\right)$. For all $g \in G$ and all $X \in E$ we have

$$
\left[R(g)\left(l_{a} \circ F\right)\right](X)=\left(l_{a} \circ F\right)(X g)=[F(X g)](a) .
$$

Also

$$
\left[l_{a} \circ D(g, \rho) F\right](X)=[(D(g, \rho) F)(X)](a)=[F(X g)](a) .
$$

Therefore $R(g)\left(l_{a} \circ F\right)=l_{a} \circ D(g, \rho) F$. This last equality implies that

$$
\Delta_{i j}\left(l_{a} \circ D(g, \rho) F\right)=\Delta_{i j}\left(R(g)\left(l_{a} \circ F\right)\right)=R(g)\left(\Delta_{i j}\left(l_{a} \circ F\right)\right) .
$$

It follows immediately from equation $(3.1)$ that $H(E, \rho)$ is invariant under the action of $G$. Therefore we can define $R(\cdot, \rho)$ as the representation of $G$ on $H(E, \rho)$ obtained by right translation. For the terminology used in the proof of the next theorem see [27], [28], and also [25].

THEOREM 3.1. Let $H(E, \xi)$ be the subspace of $S\left(E^{*}\right)$ consisting of all $G$ harmonic $\xi$-covariant polynomial functions on $E$. If $k>2 n$, then the representation $R(\cdot, \xi)$ of $G$ on $H(E, \xi)$ is irreducible and its highest weight is

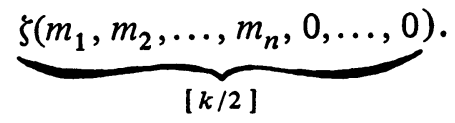

COROLlARY 3.2. If $k>2 n$, then the representation $R(\cdot, \rho)$ of $G$ on $H(E, \rho)$ is equivalent to the representation $R(\cdot, \xi)$. Hence, $R(\cdot, \rho)$ is irreducible. 
Proof OF Theorem 3.1. In what follows, we shall always divide the $k$ by $k$ complex matrices in block form as below. (If there is subsequently any exception to this, then we will expressly state the exception.)

Case 1.

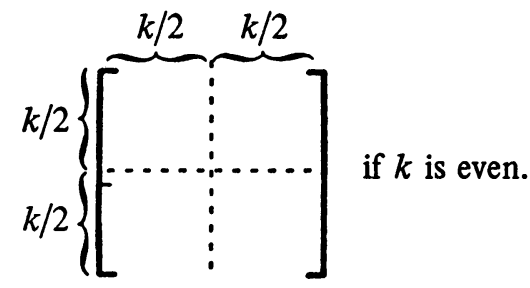

Case 2.

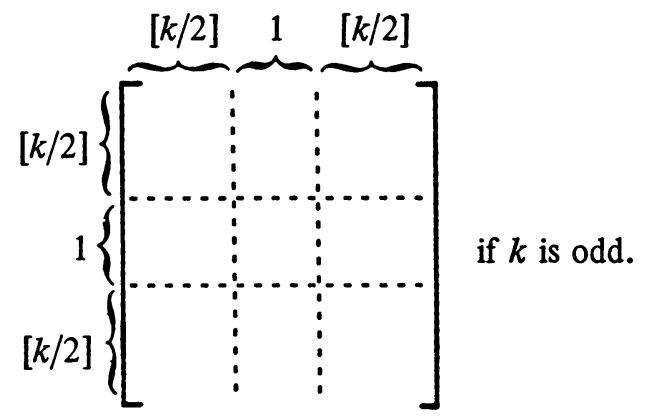

Let $q=(1 / \sqrt{2})\left[\begin{array}{cc}1 & 1 \\ i & -i\end{array}\right]$ for Case 1 ; then $q^{-1}=(1 / \sqrt{2})\left[\begin{array}{cc}1 & -i \\ 1\end{array}\right]$ and $\left(q^{-1}\right)\left(q^{-1}\right)^{t}$ $=\left[\begin{array}{ll}0 & 1 \\ 1 & 0\end{array}\right]=s$. For Case 2, we let

so that

$$
q=(1 / \sqrt{2})\left[\begin{array}{ccc}
1 & 0 & 1 \\
0 & \sqrt{2} & 0 \\
i & 0 & -i
\end{array}\right] \text { and } s=\left[\begin{array}{lll}
0 & 0 & 1 \\
0 & 1 & 0 \\
1 & 0 & 0
\end{array}\right],
$$

$$
q^{-1}=(1 / \sqrt{2})\left[\begin{array}{ccc}
1 & 0 & -i \\
0 & \sqrt{2} & 0 \\
1 & 0 & i
\end{array}\right] \text { and }\left(q^{-1}\right)\left(q^{-1}\right)^{t}=s \text {. }
$$

In any case, we let $\widetilde{G}=q^{-1} G q=\left\{\widetilde{g} \in \mathrm{GL}(k, \mathrm{C}): \widetilde{g}=q^{-1} g q, g \in G\right\}$. Then it is easy to verify that

$$
\widetilde{G}=\left\{\widetilde{g} \in \mathrm{GL}(k, \mathrm{C}): \widetilde{g} s \widetilde{g}^{t}=s, \operatorname{det} \widetilde{g}=1\right\} .
$$

Following Zhelobenko (cf. $[28$, p. 10]) we shall say that a topological group $G$ admits a Gauss decomposition (or triangular decomposition) if $C, U, V$ are closed subgroups of $G$ such that:

(i) $U$ and $V$ are normalized by $C$,

(ii) $C U$ and $C V$ are connected, closed, solvable subgroups of $G$,

(iii) $C \cap U=\{1\}$ and $C U \cap V=\{1\}$, 
(iv) $C U V$ is dense in $G$.

Now if $k$ is even we let

$$
C=\left\{c \in \mathrm{GL}(k, \mathbf{C}): c=\left[\begin{array}{cc}
b & 0 \\
0 & b^{-1}
\end{array}\right] ; b \text { an invertible diagonal matrix }\right\}
$$

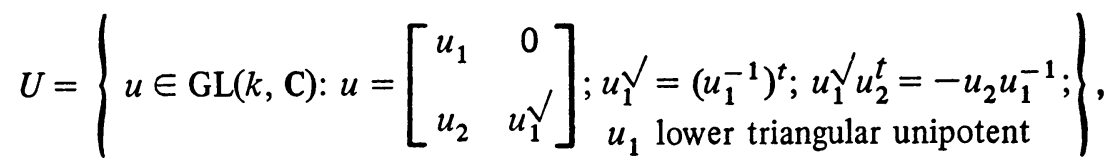

$$
\begin{aligned}
& V=\left\{v \in \mathrm{GL}(k, \mathbf{C}): v=\left[\begin{array}{cc}
v_{1} & v_{2} \\
0 & v_{1}^{\sqrt{ }}
\end{array}\right] ; \begin{array}{c}
v_{1}^{\sqrt{ }}=\left(v_{1}^{-1}\right)^{t}, v_{2} v_{1}^{t}=-v_{1} v_{2}^{t} ; \\
v_{1} \text { upper triangular unipotent }
\end{array}\right\} \text {. }
\end{aligned}
$$

If $k$ is odd we let

$$
\begin{aligned}
& C=\left\{c \in \mathrm{GL}(k, \mathbf{C}): c=\left[\begin{array}{ccc}
b & 0 & 0 \\
0 & 1 & 0 \\
0 & 0 & b^{-1}
\end{array}\right] ; b \text { an invertible diagonal matrix }\right\} \text {, } \\
& \text { (3.3) } U=\left\{u \in \mathrm{GL}(k, \mathbf{C}): u=\left[\begin{array}{ccc}
u_{1} & 0 & 0 \\
u_{2} & 1 & 0 \\
u_{3} & u_{4} & u_{1}^{\sqrt{ }}
\end{array}\right] ; \begin{array}{c}
u_{4}^{t}+u_{2} u_{1}^{-1}=0 ; \\
u_{1}^{\sqrt{ }} u_{3}^{t}+u_{4} u_{4}^{t}+u_{3} u_{1}^{-1}=0 ; \\
u_{1} \text { lower triangular unipotent }
\end{array}\right\} \text {, } \\
& V=\left\{v \in \mathrm{GL}(k, \mathbf{C}): v=\left[\begin{array}{lll}
v_{1} & v_{2} & v_{3} \\
0 & 1 & v_{4} \\
0 & 0 & v_{1}^{\sqrt{ }}
\end{array}\right] ; \begin{array}{c}
v_{4} v_{1}^{t}+v_{2}=0 ; \\
v_{3} v_{1}^{t}+v_{2} v_{2}^{t}+v_{1} v_{3}^{t}=0 ; \\
v_{1} \text { upper triangular unipotent }
\end{array}\right\} \text {. }
\end{aligned}
$$

We now proceed to prove Theorem 3.1 via several lemmas.

LEMmA 3.3. The subgroups $C, U, V$ (given by equation (3.2) for the case $k$ even, and by (3.3) for the case $k$ odd) constitute a triangular decomposition for $\widetilde{G}$.

Proof. See [27] and [28]. Notice that the Bruhat decomposition lemma may be used to prove that $C U V$ is dense in $\widetilde{G}$; also $(C, U, V)$ is a triangular decomposition of $\widetilde{G}$ induced by the triangular decomposition of the full linear group $\mathrm{GL}(k, \mathrm{C})$. Finally, remark that in [28, p. 41], Zhelobenko used the matrix

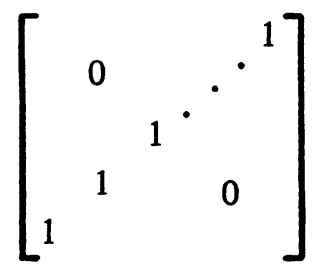

instead of the matrix $s$ used here; however this difference is inessential.

Now let $T$ be the maximal torus of $G_{0}$ (i.e. maximal connected abelian 
subgroup) consisting of all $k$ by $k$ matrices $t$ with

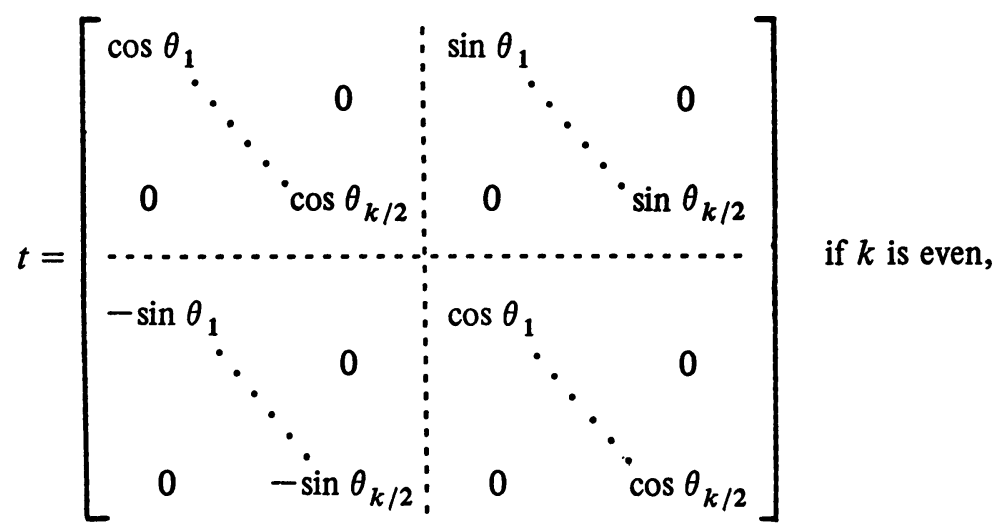

and

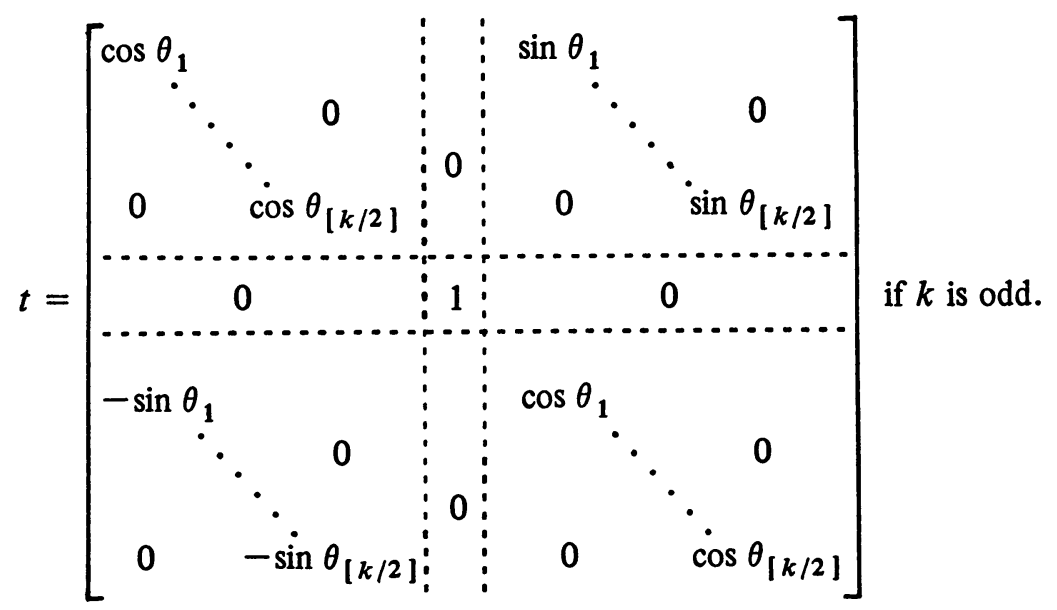

Then $\widetilde{T}=q^{-1} T q$ is a maximal torus of $\widetilde{G}_{0}$. In fact, $\widetilde{T}$ consists of all elements $\tilde{t}$ given by

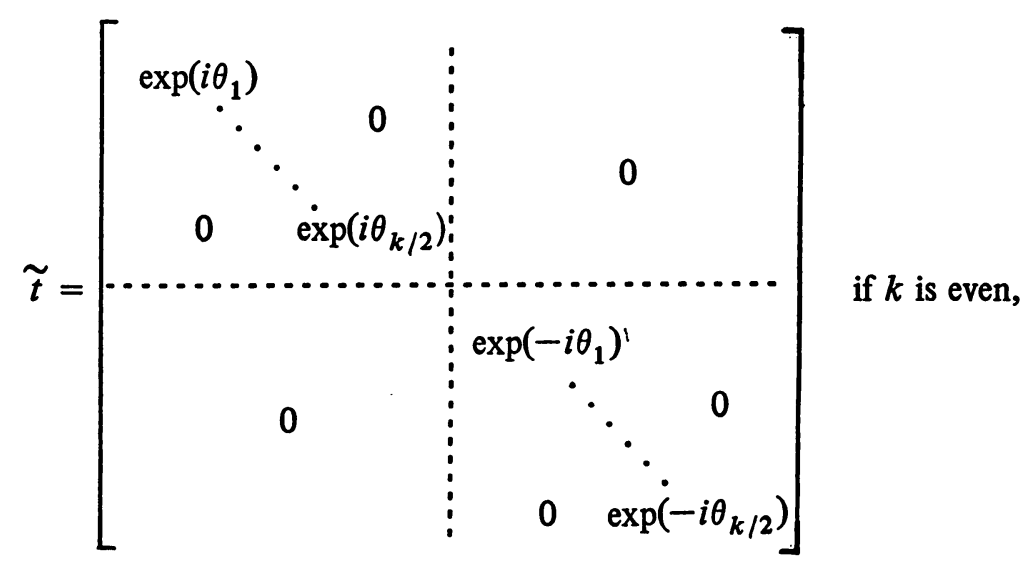


and

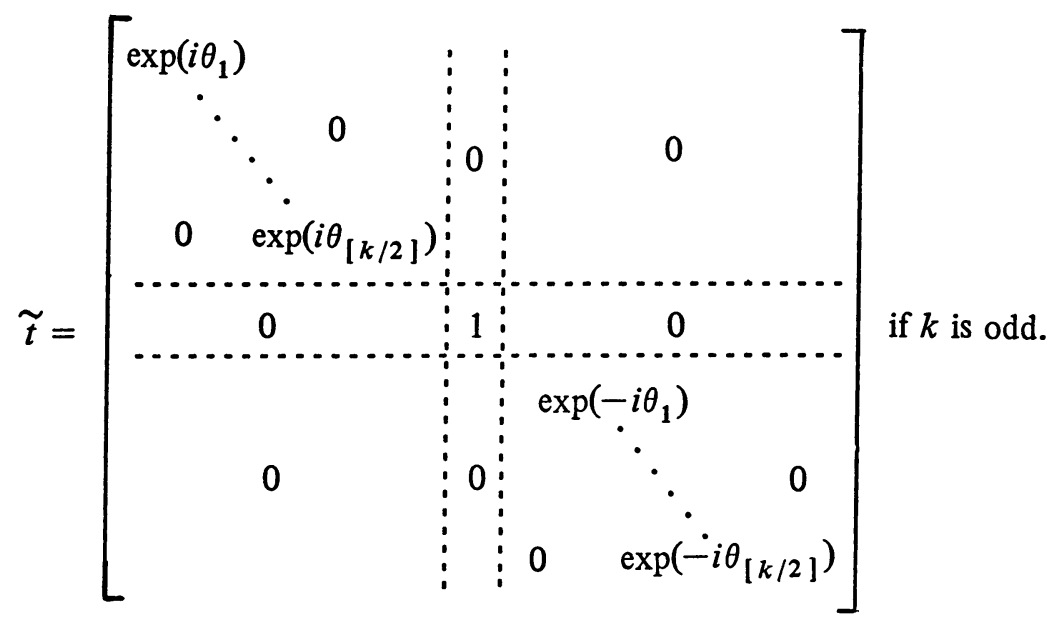

Let $\widetilde{B}=C U$; then $\widetilde{B}$ is a maximal connected solvable subgroup of $\widetilde{G}$ (i.e., a Borel subgroup of $\widetilde{G}$ ). From now on, we shall always assume that $k$ is even; the case $k$ is odd may be treated similarly.

Now let $\zeta$ be a character of $\widetilde{T}$ defined $b y$

$$
\zeta(\tilde{t})=\exp \left(i\left(m_{1} \theta_{1}+\cdots+m_{n} \theta_{n}\right)\right)
$$

the $m_{i}$ 's $(i=1, \ldots, n)$ are exactly the same as in $\xi\left(m_{1}, \ldots, m_{n}\right)$. Then $\zeta$ extends uniquely to a holomorphic character of $\widetilde{B}$, which we shall again denote by $\zeta$.

Hence

$$
\zeta(\widetilde{b})=b_{11}^{m_{1}} b_{22}^{m_{2}} \cdots b_{n n}^{m_{n}} \quad \text { if } \widetilde{b}=c u \in \widetilde{B} \text { and } c=\left[\begin{array}{cc}
b & 0 \\
0 & b^{\sqrt{ }}
\end{array}\right]
$$

Let $\operatorname{Hol}(\widetilde{G}, \zeta)$ denote the linear space of all $\zeta$-covariant holomorphic functions defined on $\widetilde{G}$. Define a representation of $G$ on $\operatorname{Hol}(\widetilde{G}, \zeta)$ by

$$
(\pi(g, \zeta) \widetilde{f})(y)=\widetilde{f}(y \widetilde{g})=\widetilde{f}\left(y q^{-1} g q\right)
$$

for all $y \in \widetilde{G}, g \in G$ and $\widetilde{f} \in \operatorname{Hol}(\widetilde{G}, \zeta)$. By the Borel-Weil-Bott theorem $\pi(\cdot, \zeta)$ is irreducible and its highest weight vector $\widetilde{f}_{\zeta}$ is given by

$$
\widetilde{f}_{\zeta}(y)=\prod_{i=1}^{n}\left[M_{i}(y)\right]^{m_{i}-m_{i+1}}
$$

where $y \in \widetilde{G}$ and $M_{i}(y)$ is the principal minor of order $i$ of the matrix $y ; i=1$, $\ldots, n$ and $m_{n+1}=0$. Now we define the function $f_{\xi}$ on $E$ by 


$$
f_{\xi}(X)=\prod_{i=1}^{n}\left[M_{i}(X)\right]^{m_{i}-m_{i+1}}
$$

For $X \in E$, set $f_{0}(X)=f_{\xi}(X q)$. Let $Z_{0} \in E$ be represented in block form as $\left[\left(I_{n}\right)(0)\left(-I_{n}^{\prime}\right)(0)\right]$ where $I_{n}$ is the unit matrix of order $n, I_{n}^{\prime}=\sqrt{-1} I_{n}$ and 0 are $n$ by $k / 2-n$ matrices with all entries equal to zero. Recall that $B$ is the lower triangular Borel subgroup of $\operatorname{GL}(n, \mathrm{C})$ and set $W=B Z_{0} G$. If $f \in H(E, \xi)$ we shall denote by $f / W$ the restriction of $f$ to $W$. Set

$$
H(W, \xi)=\{f / W: f \in H(E, \xi)\} .
$$

Define the representation $T(\cdot, \xi)$ of $G$ on $H(W, \xi)$ by

$$
[T(g, \xi)(f / W)](w)=f(w g)
$$

for all $(w, g) \in W \times G$ and all $f / W \in H(W, \xi)$. Next we shall prove

LEMmA 3.4. The function $f_{0}$ is $G$-harmonic and $\xi$-covariant, i.e., $f_{0} \in$ $H(E, \xi)$.

Proof. It is easy to verify that $f_{0}$ is $\xi$-covariant from the explicit expression of $f_{0}$. It remains to show that $f_{0}$ is $G$-harmonic, and for this is it sufficient to show that $\Delta_{i j} f_{0}=0$ for all $i, j, 1 \leqslant i \leqslant j \leqslant n$. If $p=k / 2$ then we have

$$
\begin{aligned}
{\left[\Delta_{i j} f_{0}\right](X) } & =\sum_{t=1}^{k}\left[D_{i t} D_{j t} f_{0}\right](X) \\
& =\sum_{r=1}^{p}\left(\left[D_{i r} D_{j r} f_{0}\right](X)+\left[D_{i, r+p} D_{j, r+p} f_{0}\right](X)\right) .
\end{aligned}
$$

Now

$$
f_{0}(X)=\prod_{h=1}^{n}\left[M_{h}(X q)\right]^{m_{h}-m_{h+1}} .
$$

Therefore, by the product rule for differentiation we have

$$
\begin{aligned}
D_{i r} D_{j r} f_{0}(X) & \\
= & \sum_{s=1}^{n}\left(D_{i r} D_{j r}\left[M_{s}(X q)\right]^{m_{s}-m_{s+1}}\right) \prod_{h=1 ;(h \neq s)}^{n}\left[M_{h}(X q)\right]^{m_{h}-m_{h+1}} \\
& +\sum_{\substack{s, t=1 \\
(s \neq t)}}^{n}\left(D_{i r}\left[M_{t}(X q)\right]^{m_{t}-m_{t+1}} D_{j r}\left[M_{s}(X q)\right]^{\left.m_{s}-m_{s+1}\right)}\right. \\
& \prod_{\substack{h=1 \\
(h \neq s, h \neq t)}}^{n}\left[M_{h}(X q)\right]^{m_{h-h+1}}
\end{aligned}
$$




$$
\begin{aligned}
& D_{i, r+p} D_{j, r+p} f_{0}(X) \\
& =\sum_{s=1}^{n}\left(D_{i, r+p} D_{j, r+p}\left[M_{s}(X q)\right]^{m_{s}-m_{s+1}}\right) \prod_{\substack{h=1 \\
(h \neq s)}}^{n}\left[M_{h}(X q)\right]^{m_{h}-m_{h+1}} \\
& +\sum_{\substack{s, t=1 \\
(s \neq t)}}^{n}\left(D_{i, r+p}\left[M_{t}(X q)\right]^{m_{t}-m_{t+1}} D_{j, r+p}\left[M_{s}(X q)\right]^{m_{s}-m_{s+1}}\right) \\
& \text { - } \prod_{\substack{n=1 \\
(h \neq s, h \neq t)}}^{n}\left[M_{h}(X q)\right]^{m_{h}-M_{h+1}} \text {. }
\end{aligned}
$$

By using the rule of differentiation for determinants it is easy to see that

$$
D_{i r} D_{j r}\left[M_{s}(X q)\right]^{m_{s}-m_{s+1}}=-D_{i, r+p} D_{j, r+p}\left[M_{s}(X q)\right]^{m_{s}-m_{s+1}}
$$

and

$$
\begin{aligned}
& D_{i r}\left[M_{t}(X q)\right]^{m_{t}-m_{t+1}} D_{j r}\left[M_{s}(X q)\right]^{m_{s}-m_{s+1}} \\
& \quad=-D_{i, r+p}\left[M_{t}(X q)\right]^{m_{t} t^{-m_{t+1}} D_{j, r+p}\left[M_{s}(X q)\right]^{m_{s}-m_{s+1}}}
\end{aligned}
$$

for every $s, t ; 1 \leqslant s, t \leqslant n \quad(s \neq t)$. Lemma 3.4 now follows immediately from (3.6), (3.7), (3.8), (3.9) and (3.10).

LEMmA 3.5. The representations $T(\cdot, \xi)$ and $\pi(\cdot, \zeta)$ of $G$ on $H(W, \xi)$ and Hol $(\widetilde{G}, \zeta)$, respectively, are equivalent. Hence, $T(\cdot, \xi)$ is irreducible and its highest weight is indexed by

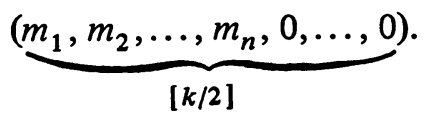

Proof. Let $b_{0}=(1 / \sqrt{2}) I_{n}$ and $I=b_{0} Z_{0} q=\left[\left(I_{n}\right)(0)\right]$. Define a map $\Lambda$ from $H(W, \xi)$ to $\operatorname{Hol}(\tilde{G}, \zeta)$ by

$$
[\Lambda(f / W)](y)=f\left(\mathbf{I} y q^{-1}\right)
$$

for all $f / W \in H(W, \xi)$ and all $y \in \widetilde{G}$. Since $\mathbf{I} y q^{-1}=b_{0} Z_{0} q y q^{-1}$ and $q y q^{-1} \in$ $G, \mathbf{I} y q^{-1} \in W$ for all $y \in \widetilde{G}$. Now if $\widetilde{b} \in \widetilde{B}, \widetilde{b}$ can be represented in block form as $\left[\begin{array}{ll}b & 0 \\ e & f\end{array}\right]$ where $b \in B$, so that $\zeta(\widetilde{b})=\xi(b)$. Now, it is clear that $\mathbf{I} \widetilde{b}=b \mathbf{I}$; hence,

$$
\begin{aligned}
{[\Lambda(f / W)](\widetilde{b} y) } & =f\left(\mathbf{I} \tilde{b} y q^{-1}\right)=f\left(b \mathbf{I} y q^{-1}\right) \\
& =\xi(b) f\left(\mathbf{I} y q^{-1}\right)=\zeta(\widetilde{b})[\Lambda(f / W)](y),
\end{aligned}
$$


and it is clear that $\Lambda(f / W)$ is a holomorphic function on $\widetilde{G}$; hence, $\Lambda$ is a welldefined map. It is also clear that $\Lambda$ is linear. Next we shall show that $\Lambda$ intertwines $T(\cdot, \xi)$ and $\pi(\cdot, \zeta)$. For all $g \in G, y \in \widetilde{G}$ and $f / W \in H(W, \xi)$ we have

$$
[\Lambda(T(g, \xi)(f / W))](y)=f\left(\mathrm{I} y q^{-1} g\right)
$$

and

$$
\begin{aligned}
{[\pi(g, \zeta) \Lambda(f / W)](y) } & =[\Lambda(f / W)](y \tilde{g}) \\
& =[\Lambda(f / W)]\left(y q^{-1} g q\right)=f\left(\mathbf{I} y q^{-1} g\right)
\end{aligned}
$$

so that

$$
\Lambda T(g, \xi)(f / W)=\pi(g, \zeta) \Lambda(f / W)
$$

for all $f / W \in H(W, \xi)$.

Now, let us show that $\Lambda$ is injective. Indeed, if $[\Lambda(f / W)](y)=f\left(b_{0} Z_{0} q y q^{-1}\right)$ $=0$ for all $y \in \widetilde{G}$, then $f\left(b_{0} Z_{0} g\right)=0$ for all $g \in G$. Since $f$ is $\xi$-covariant, this implies immediately that $f(w)=0$ for all $w \in W$; i.e., $f / W=0$.

By a simple computation we see that

$$
\left[\Lambda\left(f_{0} / W\right)\right](y)=f_{0}\left(\mathbf{I} y q^{-1}\right)=f_{\xi}(\mathbf{I} y)=f_{\zeta}(y) .
$$

Hence $\Lambda(H(W, \xi))$ is nonzero; and since $\pi(\cdot, \zeta)$ is irreducible, it follows immediately that $\Lambda$ is bijective. This fact completes the proof of Lemma 3.5.

Now since $k>2 n$, there exists a permutation matrix $g$ belonging to $G_{0}$ such that $X_{0} g=Z_{0}$ (recall that $X_{0}=\left[\left(I_{n}\right)\left(-I_{n}^{\prime}\right)(0)\right]$ as defined in $\left.\S 2\right)$. Therefore $W=B Z_{0} G=B X_{0} G$. In fact, it is easy to see that $W=X_{0} G=P_{n}$. Now by Corollary 2.7 it is clear that the restriction mapping $f \rightarrow f / W$ of $H(E, \xi)$ onto $H(W, \xi)$ is a $G$-module isomorphism. This fact and Lemma 3.5 immediately imply Theorem 3.1.

Proof of Corollary 3.2. Recall that the mapping $\Phi$ from $P(E, \xi)$ onto $P(E, \rho)$ as defined in Theorem 1.5 was given by $[(\Phi f)(X)](a)=f(a X)$ for all $a \in \mathrm{GL}(n, \mathrm{C}), X \in E$, and $f \in P(E, \xi)$. The map $\Phi$ is actually an isomorphism and its inverse mapping is given by $\left(\Phi^{-1} F\right)(X)=(F(X))(1)$ for all $F \in P(E, \rho)$. Now to prove Corollary 3.2 it is sufficient to verify that the restriction of $\Phi$ to $H(E, \xi)$ is an isomorphism between $H(E, \xi)$ and $H(E, \rho)$, since it is evident from Theorem 1.5 that $\Phi / H(E, \xi)$ intertwines $R(\cdot, \xi)$ and $R(\cdot, \rho)$. First let us show that $\Phi^{-1}(H(E, \rho)) \subset H(E, \xi)$. Indeed, if $F \in H(E, \rho)$ then $\Delta_{i j}\left(l_{a} \circ F\right)=0$ for all $a \in \mathrm{GL}(n, \mathrm{C}), 1 \leqslant i \leqslant j \leqslant n$; in particular, $\Delta_{i j}\left(l_{1} \circ F\right)=0$. But $\left(\Phi^{-1} F\right)(X)$ $=(F(X))(1)=\left(l_{1} \circ F\right)(X)$ for all $X \in E$. Hence, $\Delta_{i j}\left(\Phi^{-1} F\right)=0$ for all $F \in$ $H(E, \rho)$ and for all $i, j, 1 \leqslant i \leqslant j \leqslant n$. Thus $\Phi^{-1} F \in H(E, \xi)$ for all $F \in H(E, \rho)$. However, this is not sufficient to conclude that $\Phi^{-1} / H(E, \rho)$ is surjective, since $H(E, \rho)$ might reduce to $\{0\}$. Instead of showing that $H(E, \rho)$ is nonzero, we prove that $\Phi(H(E, \xi)) \subset H(E, \rho)$. For this purpose, we suppose that $f \in H(E, \xi)$ 
and verify that $\Delta_{i j}\left(l_{a} \circ \Phi f\right)=0$ for all $a \in \operatorname{GL}(n, \mathrm{C})$ and all $i, j, 1 \leqslant i \leqslant j \leqslant n$.

For $a \in \mathrm{GL}(n, \mathrm{C})$, let $L(a)$ denote the linear map $X \rightarrow a X, X \in E$. Then

$$
[f \circ L(a)](X)=f(a X)=[(\Phi f)(X)](a)=\left[l_{a} \circ \Phi f\right](X) .
$$

Hence, $f \circ L(a)=l_{a} \circ \Phi f$, and $\Delta_{i j}\left(l_{a} \circ \Phi f\right)=\Delta_{i j}(f \circ L(a))$. Setting $Y=a X$ and using the chain rule for differentiation, we get

$$
\left[D_{j t}(f \circ L(a))\right](X)=\sum_{s=1}^{n} a_{s j}\left[D_{s t} f\right](Y) .
$$

Thus

$$
\left[D_{i t} D_{j t}(f \circ L(a))\right](X)=\sum_{r, s=1}^{n} a_{r i} a_{s j}\left[D_{r t} D_{s t} f\right](Y)
$$

and so

$$
\begin{aligned}
{\left[\Delta_{i j}(f \circ L(a))\right](X) } & =\sum_{t=1}^{n}\left[D_{i t} D_{j t}(f \circ L(a))\right](X) \\
& =\sum_{t=1}^{k} \sum_{r, s=1}^{n} a_{r i} a_{s j}\left[D_{r t} D_{s t} f\right](Y) \\
& =\sum_{r, s=1}^{n} a_{r i} a_{s j}\left\{\sum_{t=1}^{k}\left[D_{r t} D_{s t} f\right](Y)\right\} \\
& =\sum_{r, s=1}^{n} a_{r i} a_{s j}\left[\Delta_{r s} f\right](Y) .
\end{aligned}
$$

Since $f \in H(E, \xi),\left[\Delta_{r s} f\right](Y)=0$ for all $Y \in E$ and all $r, s, 1 \leqslant r \leqslant s \leqslant n$. From equations (3.12) and (3.14) we infer that $\Delta_{i j}\left(l_{a} \circ \Phi f\right)=0$ for all $i, j, 1 \leqslant i \leqslant$ $j \leqslant n$.

REMARKS 3.6. (1) As in Note 2.6 we can easily see that $H(E, \xi)$ is a subspace of $H^{m}\left(E^{*}\right)$, the linear space of all harmonic homogeneous polynomials of degree $m$ on $E$.

(2) After Lemma 3.5 we could also complete the proof of Theorem 3.1 by invoking Theorem 1.3 of [12] and by observing that the space of all $G$-harmonic polynomials on $E$ which vanish identically on $P$ is reduced to $\{0\}$.

(3) A particularly interesting class of $\xi$-covariant polynomials on $E$ arises in the special case where all the $m_{i}(1 \leqslant i \leqslant n)$ in $\xi\left(m_{1}, m_{2}, \ldots, m_{n}\right)$ are equal to a fixed integer $m \geqslant 0$. Thus when $\xi=\xi(m, m, \ldots, m)$ we shall denote $H(E, \xi)$ by $H(E, m)$. Now, following C. Herz (cf. [13, p. 482]) we shall say that a polynomial function $f$ on $E$ is determinantly homogeneous of degree $m$ if $f(a X)$ $=(\operatorname{det} a)^{m} f(X)$ for all $a \in \mathrm{GL}(n, \mathbf{C})$. In this context we have

COROllary 3.7. For every $\xi=(m, m, \ldots, m)$ the simple $G$-module $H(E, m)$ consists of all determinantly homogeneous G-harmonic polynomials of 
degree $m$ on $E$. Moreover, $H(E, m)$ is spanned by the particular harmonics $f$ of the form

$$
f(X)=\left(\operatorname{det}\left(L X^{t}\right)\right)^{m}, \quad L, X \in E \text { and } L L^{t}=0 .
$$

Proof. From Theorem 1.5, we see that if $\xi=\xi(m, m, \ldots, m)$, then the highest weight vector $f_{\xi}$ of $P(E, \xi)=P(E, m)$ is given by $f_{\xi}(X)=\left(\operatorname{det}\left(X_{1}\right)\right)^{m}$ where $X \in E$ is represented in block form as $\left[X_{1} X_{2}\right]$, with $X_{1}$ an $n \times n$ matrix. Since $P(E, m)$ is the $G$-invariant subspace spanned by $f_{\xi}$, it is easy to verify that $P(E, m)$ consists precisely of all determinantly homogeneous polynomials of degree $m$ on $E$. At this point, we recall that $f_{0}=D(q, \xi) f_{\xi}$ belongs to $H(E, m)$. Because $R(\cdot, \xi)=R(\cdot, m)$ is an irreducible representation of $G$ on $H(E, m)$, the polynomial functions $R(g, m) f_{0}$ span $H(E, m)$. Let $L_{0}^{t}$ be the first $k \times n$ block of the matrix $q$. Clearly $L_{0} L_{0}^{t}=0$; therefore $f_{0}(X)=\left(\operatorname{det}\left(X L_{0}^{t}\right)\right)^{m}$ and

$$
\left(R(g, m) f_{0}\right)(X)=\left(\operatorname{det}\left(X g L_{0}^{t}\right)\right)^{m}=\left(\operatorname{det}\left(X g L_{0}^{t}\right)^{t}\right)^{m}=\left(\operatorname{det}\left(\left(L_{0} g^{t}\right) X^{t}\right)\right)^{m} .
$$

Set $L_{0} g^{t}=L$, then $L L^{t}=\left(L_{0} g^{t}\right)\left(g L_{0}^{t}\right)=L_{0} L_{0}^{t}=0$.

Now recall that $G_{0}$ is the real special orthogonal subgroup of order $k$, let $U(k)$ denote the unitary subgroup of $\operatorname{GL}(k, \mathrm{C})$, and $D_{0}(\cdot, \rho)\left(\operatorname{resp} . D_{0}(\cdot, \xi)\right)$ denote the restriction of $D(\cdot, \rho)$ (resp. $D(\cdot, \xi)$ ) to $U(k)$. Then, by Theorem $1.1, D_{0}(\cdot, \rho)$ and $D_{0}(\cdot, \xi)$ are irreducible. Similarly, $R_{0}(\cdot, \rho)=R(\cdot, \rho) / G_{0}$ and $R_{0}(\cdot, \xi)=R(\cdot, \xi) / G_{0}$ are equivalent irreducible representations of $G_{0}$. From $\S 1$, and the first part of this section, it follows that the $\rho$-covariant and $\xi$-covariant polynomials on $E$ are intimately related. In the proof of Theorem 1.3, we used the irreducibility of $D(\cdot, \xi)$, indirectly, via Theorem 1.5 , to see that $D(\cdot, \rho)$ is irreducible. Actually it may be shown directly that $D(\cdot, \rho)$ is irreducible. Indeed, let $P$ denote the parabolic subgroup of $\operatorname{GL}(k, \mathbf{C})$ consisting of all matrices given in block form as $\left[\begin{array}{cc}a & 0 \\ c & d\end{array}\right]$ with $a \in \operatorname{GL}(n, \mathbf{C})$ and set $\tilde{\rho}\left(\left[\begin{array}{ll}a & 0 \\ c & d\end{array}\right]\right)=$ $\rho(a)$. Then we may apply the inducing technique of Theorem 1.1 to $P$ and easily show that $D(\cdot, \rho)$ is equivalent to the irreducible holomorphic induced representation $\pi(\cdot, \tilde{\rho})$ of $\mathrm{GL}(k, \mathbf{C})$.

In the sequel, we shall reverse the scheme used in $\S 1$ and previously in this section, i.e., we shall assume that $D_{0}(\cdot, \rho)$ and $R_{0}(\cdot, \rho)$ are irreducible and then prove that $D_{0}(\cdot, \xi)$ and $R_{0}(\cdot, \xi)$ are irreducible. This approach gives interesting results which subsequently will be used in $\S 4$. However, instead of achieving these results in that particular context, we shall attain them in a more general setting.

Let $E$ be a finite dimensional complex vector space. Let $U$ and $K$ be two compact Lie groups. Suppose that $U$ and $K$ act linearly on $E$ to the left and to the right respectively; moreover, we assume that the two actions commute. Let $\rho$ be a continuous irreducible unitary representation of $U$ on a complex Hilbert space $W$. Suppose that we are given a nonzero finite dimensional complex vector 
space $H_{\rho}$ which consists of $\rho$-covariant mappings from $E$ into $W$. We also require that the representation $T$ of $K$ obtained by right translation on $H_{\rho}$ is irreducible. The commutativity of the two actions (of $U$ and $K$, respectively, on $E$ ) insures that $H_{\rho}$ is $T$-invariant.

Let $W^{*}$ denote the dual of $W$ and set

$$
\tilde{H}_{\rho}=\left\{l \circ F: l \in W^{*}, F \in H_{\rho}\right\} .
$$

Then clearly $\widetilde{H}_{\rho}$ is a finite dimensional complex vector space. Next, let $\widetilde{T}$ be the representation of $K$ on $\tilde{H}_{\rho}$ defined by:

$$
\begin{aligned}
{[\tilde{T}(g)(l \circ F)](X)=} & {[l \circ F](X g)=l(F(X g)) } \\
= & l(T(g) F(X))=[l \circ(T(g) F)](X) \\
& \text { for all } l \in W^{*}, F \in H_{\rho}, g \in K \text { and } X \in E .
\end{aligned}
$$

Now we make the additional assumption that $H_{\rho}$ and $\tilde{H}_{\rho}$ are equipped with appropriate inner products in which $T$ and $\widetilde{T}$ are continuous unitary representations of $K$. Let $d_{\rho}$ and $d_{T}$ denote the degrees of $\rho$ and $T$ respectively. Under the above hypotheses we have

THEOREM 3.8. The Hilbert space $\tilde{H}_{\rho}$ may be decomposed into an orthogonal direct sum of $\widetilde{T}$-invariant subspaces, i.e.,

$$
\tilde{H}_{\rho}=\sum_{\alpha=1}^{d_{\rho}} \oplus\left(\tilde{H}_{\rho}\right)_{\alpha}
$$

in such a way that, for every $\alpha=1,2, \ldots, d_{\rho}$, if $\widetilde{T}_{\alpha}$ denotes the subrepresentation of $\widetilde{T}$ on $\left(\widetilde{H}_{\rho}\right)_{\alpha}$, then $\widetilde{T}_{\alpha}$ is unitary equivalent to $T$.

Proof. Let $\langle\cdot, \cdot\rangle$ and $(\cdot, \cdot)$ denote the given inner products on $W$ and $H_{\rho}$ respectively. Let $\left\{\varphi_{1}, \varphi_{2}, \ldots, \varphi_{d_{\rho}}\right\}$ be an orthonormal basis for $W$, and let $\left\{l^{1}, l^{2}, \ldots, l^{d} \rho\right\}$ denote its dual basis. Let $\left\{F_{1}, F_{2}, \ldots, F_{d_{T}}\right\}$ be an orthonormal basis for $H_{\rho}$.

Set

$$
\begin{array}{ll}
\rho(u)_{r s}=\left\langle\rho(u) \varphi_{s}, \varphi_{r}\right\rangle & \text { for all } u \in U ; r, s=1,2, \ldots, d_{\rho} \\
T(g)_{i j}=\left(T(g) F_{j}, F_{i}\right) & \text { for all } g \in K ; i, j=1,2, \ldots, d_{T}
\end{array}
$$

Consider the subspace $l^{j}\left(H_{\rho}\right)=\left\{l^{j} \circ F: F \in H_{\rho}\right\}$; then clearly $l^{j}\left(H_{\rho}\right)$ is spanned by the functions $F_{i}^{j}=l^{\dot{j}} \circ F_{i}\left(1 \leqslant i \leqslant d_{T}\right)$; moreover, $l^{j}\left(H_{\rho}\right)$ is a $\widetilde{T}$-in- 
variant subspace of $\widetilde{H}_{\rho}$ for every fixed $j, j=1, \ldots, d_{\rho}$. Indeed, from (3.16) we get

$$
\widetilde{T}(g)\left(l^{j} \circ F\right)=l^{j} \circ(T(g) F)
$$

and since $T(g) F$ belongs to $H_{\rho}, \widetilde{T}(g)\left(l^{j} \circ F\right)$ belongs to $l^{j}\left(H_{\rho}\right)$.

Let $\widetilde{T}\left(\cdot, l^{j}\right)$ denote the restriction of $\widetilde{T}$ to $l^{j}\left(H_{\rho}\right)$. For every $j$, define a linear map $\Phi_{j}$ from $H_{\rho}$ onto $l^{j}\left(H_{\rho}\right)$ by

$$
\Phi_{j}(F)=l^{j} \circ F \quad \text { for all } F \in H_{\rho} .
$$

From equation (3.16), it is clear that each $\Phi_{j}$ interwines $T$ and $\widetilde{T}\left(\cdot, l^{j}\right)$. Because $T$ is irreducible $\Phi_{j}$ is either injective or identically zero. We shall prove that for every $j=1, \ldots, d_{\rho}, \Phi_{j}$ is actually a $K$-module isomorphism. For this purpose, we shall prove

LEMMA 3.9. The system $\left\{F_{i}^{j}\right\}_{i j}, 1 \leqslant j \leqslant d_{\rho}, 1 \leqslant i \leqslant d_{T}$, constitutes $a$ basis for $\widetilde{H}_{\rho}$.

PRoof. For every $F \in H_{\rho}$ and every $l \in W^{*}$ we write $F=\Sigma_{i=1}^{d} a_{i} F_{i}$, and $l=\Sigma_{j=1}^{d} \rho b_{j} l^{j}$. Hence

$$
l \circ F=\sum_{i j} a_{i} b_{j}\left(l^{j} \circ F_{i}\right)=\sum_{i j} a_{i} b_{j} F_{i}^{j},
$$

and this implies that the system $\left\{F_{i}^{j}\right\}_{i j}$ generates $\widetilde{H}_{\rho}$. Now suppose that $\Sigma_{i, j} c_{i j} F_{i}^{j}=0$ for $c_{i j} \in \mathbf{C}, 1 \leqslant i \leqslant d_{T}, 1 \leqslant j \leqslant d_{\rho}$. From equation (3.17) we get

$$
T(g) F_{i}=\sum_{t=1}^{d_{T}} T(g)_{t i} F_{t} \text { for } i=1, \ldots, d_{T} ; g \in K .
$$

Therefore, by equation (3.16) we obtain

$$
\begin{aligned}
{\left[\widetilde{T}(g) F_{i}^{j}\right](X) } & =\left[l^{j} \circ\left(T(g) F_{i}\right)\right](X)=l^{j}\left(\sum_{t=1}^{d} T(g)_{t i} F_{t}(X)\right) \\
& =\sum_{t=1}^{d_{T}} T(g)_{t i}\left(l^{j} \circ F_{t}\right)(X)=\sum_{t=1}^{d} T(g)_{t i} F_{t}^{j}(X) .
\end{aligned}
$$

Next, for $u \in U$, and $X \in E$ we have

and

$$
\rho(u) \varphi_{s}=\sum_{r=1}^{d} \rho(u)_{r s} \varphi_{r}
$$




$$
\begin{aligned}
F_{i}(u X) & =\rho(u) F_{i}(X)=\rho(u) \sum_{s=1}^{d_{\rho}}\left(F_{i}^{s}(X) \varphi_{s}\right) \\
& =\sum_{s=1}^{d_{\rho}} F_{i}^{s}(X) \rho(u) \varphi_{s}=\sum_{s=1}^{d_{\rho}} F_{i}^{s}(X)\left(\sum_{j=1}^{d_{\rho}} \rho(u)_{j s} \varphi_{j}\right) \\
& =\sum_{j=1}^{d_{\rho}}\left(\sum_{s=1}^{d_{\rho}} \rho(u)_{j s} F_{i}^{s}(X)\right) \varphi_{j} .
\end{aligned}
$$

Therefore, from equations (3.18) and (3.19) we get

$$
\begin{aligned}
& F_{i}^{j}(u X g)= \sum_{s=1}^{d_{\rho}} \rho(u)_{j s} F_{i}^{s}(X g)=\sum_{s=1}^{d_{\rho}} \rho(u)_{j s}\left[\widetilde{T}(g) F_{i}^{s}\right](X) \\
&= \sum_{s=1}^{d_{\rho}} \rho(u)_{j s} \sum_{t=1}^{d_{T}} T(g)_{t i} F_{t}^{s}(X)=\sum_{s, t} \rho(u)_{j s} T(g)_{t i} F_{t}^{s}(X) ; \\
& 1 \leqslant s \leqslant d_{\rho} ; 1 \leqslant t \leqslant d_{T} .
\end{aligned}
$$

By assumption we have

$$
\sum_{i, j} c_{i j} F_{i}^{j}(u X g)=0 ; \quad 1 \leqslant i \leqslant d_{T} ; 1 \leqslant j \leqslant d_{\rho},
$$

$$
\text { for all } u \in U, X \in E \text {, and } g \in K \text {. }
$$

Recall that from Schur's orthogonality relations we have

$$
d_{\rho} \int_{U} \rho(u)_{j s}{\overline{\rho(u)_{\alpha \beta}}} d u=\delta_{j \alpha} \delta_{s \beta} \quad \text { and } \quad d_{T} \int_{K} T(g)_{t i} \overline{T(g)}_{\gamma \eta} d g=\delta_{t \gamma} \delta_{i \eta} \text {, }
$$

where $\delta_{i j}$ are the Kronecker symbols and $d u, d g$ are normalized Haar measures on $U$ and $K$ respectively.

From equations (3.20) and (3.21) we infer that

$$
\begin{aligned}
0 & =d_{\rho} d_{T} \int_{U \times K} \overline{\rho(u)_{\alpha \beta}} \bar{T}(g)_{\gamma \eta}\left(\sum_{i j} c_{i j} F_{i}^{j}(u X g)\right) d u d g \\
& =d_{\rho} d_{T} \int_{U \times K} \overline{\rho(u)_{\alpha \beta}} \bar{T}(g)_{\gamma \eta}\left\{\sum_{i j} c_{i j}\left(\sum_{s, t} \rho(u)_{j s} T(g)_{t i} F_{t}^{s}(X)\right)\right\} d u d g \\
& =d_{\rho} d_{T} \sum_{i, j} c_{i j}\left\{\sum_{s, t} F_{t}^{s}(X)\left(\int_{U \times K} \rho(u)_{j s} \overline{\rho(u)_{\alpha \beta}} T(g)_{t i} \overline{T(g)}{ }_{\gamma \eta} d u d g\right)\right\} \\
& =d_{\rho} d_{T} \sum_{i, j} c_{i j}\left\{\sum_{s, t} F_{t}^{s}(X)\left(\int_{U \times K} \rho(u)_{j s} \overline{\rho(u)_{\alpha \beta}} d u\right)\left(\int_{K} T(g)_{t i} \overline{T(g)_{\gamma \eta}} d g\right)\right\} \\
& =\sum_{i, j} c_{i j}\left\{\sum_{s, t} F_{t}^{s}(X) \delta_{j \alpha} \delta_{s \beta} \delta_{t \gamma} \delta_{i \eta}\right\}=\sum_{i, j} c_{i j} F_{\gamma}^{\beta}(X) \delta_{j \alpha} \delta_{i \eta} \\
& =c_{\eta \alpha} F_{\gamma}^{\prime \beta}(X) \quad \text { for all } \eta, \alpha, \beta, \gamma \text { and all } X \in E ; 1 \leqslant \eta, \gamma \leqslant d_{T}, 1 \leqslant \alpha, \beta \leqslant d_{\rho} .
\end{aligned}
$$


This implies immediately that $c_{i j}=0$ for all $i, j\left(1 \leqslant i \leqslant d_{T}, 1 \leqslant j \leqslant d_{\rho}\right)$. Hence, the proof of Lemma 3.9 is concluded.

Now, by construction, each $\Phi_{j}$ maps $H_{\rho}$ onto $l^{j}\left(H_{\rho}\right)$, and Lemma 3.9 shows that $\operatorname{dim}\left(H_{\rho}\right)=\operatorname{dim}\left(l^{j}\left(H_{\rho}\right)\right)$. Hence, $\Phi_{j}$ is a $K$-module isomorphism. By a well-known fact (see, e.g., [20, p. 285]) we may assume that each $\widetilde{T}\left(\cdot, l^{j}\right)$ is unitary equivalent to $T$. This implies that the representations $\widetilde{T}\left(\cdot, l^{j}\right), 1 \leqslant j \leqslant$ $d_{\rho}$, are unitary equivalent to each other.

As continuous unitary representation of a compact Lie group, $\widetilde{T}$ decomposes completely into a sum of continuous irreducible unitary subrepresentations $\widetilde{T}_{\alpha}$, $\alpha=1,2, \ldots$ Therefore $\widetilde{H}_{\rho}$ may be represented as an orthogonal direct sum of its subspaces.

$$
\tilde{H}_{\rho}=\sum_{\alpha} \bigoplus\left(\tilde{H}_{\rho}\right)_{\alpha} ; \quad \tilde{T} /\left(\tilde{H}_{\rho}\right)_{\alpha}=\tilde{T}_{\alpha} .
$$

Now by the above paragraph, as $K$-module $\widetilde{H}_{\rho}$ is also a direct sum (not necessarily orthogonal) of the submodules $l^{j}\left(H_{\rho}\right)$. By a classical result concerning decompositions of semisimple $K$-modules (cf., e.g., [5, Proposition 3, p. 175]), we may conclude that the $\widetilde{T}_{\alpha}$ in equation (3.22) are unitary equivalent to each other and that $1 \leqslant \alpha \leqslant d_{\rho}$.

Now we recall from $\S 2$ that $S\left(E^{*}\right)$ was equipped with the inner product defined by $(p, q)=(p(D) \tilde{q})(0)$. For $y \in \operatorname{GL}(k, \mathbf{C})$ we remark that $(R(y) q)^{\sim}$ $=R(\bar{y}) \tilde{q}$. Therefore, using equation (2.1) we get

$$
(R(y) p, R(y) q)=\left(R(y) p(D) R\left(y^{t}\right) R(\bar{y}) \widetilde{q}\right)(0)
$$

If $y \in U(k)$ or $y \in O(k)$, then $y^{t} \bar{y}=1$, and in that case, by using equation (3.23), we have $(R(y) p, R(y) q)=(p, q)$.

Choose an orthonormal basis $\left\{\varphi_{1}, \varphi_{2}, \ldots, \varphi_{d_{\rho}}\right\}$ for $\operatorname{Hol}(\mathrm{GL}(n, \mathrm{C}), \xi)$ in such a manner that the first element $l^{1}$ of its dual basis $\left\{l^{1}, l^{2}, \ldots, l^{d} \rho\right\}$ satisfies $l^{1}(\varphi)=c \varphi(1)$ for all $\varphi \in \operatorname{Hol}(\operatorname{GL}(n, \mathbf{C})$, $\xi)$, where $c$ is a constant of normalizations. Let $\rho_{0}$ denote the restriction of $\rho$ to $U(n)$; since $U(n)$ is compact we may assume, without loss of generality, that $\rho_{0}$ is a continuous irreducible unitary representation. Set

$$
\left.(P(E, \rho))^{\sim}=\{l \circ F: F \in P(E, \rho), l \in(H \circ \mathrm{GL}(n, \mathrm{C}), \xi))^{*}\right\}
$$

then clearly $(P(E, \rho))^{\sim}$ is a subspace of $S\left(E^{*}\right)$. Let $\widetilde{D}_{0}(\cdot, \rho)$ denote the representation of $U(k)$ obtained by right translation on $(P(E, \rho))^{\sim}$. The above inner product on $S\left(E^{*}\right)$ induces a Hilbert space structure on $(P(E, \rho))^{\sim}$ such that $\widetilde{D}_{0}(\cdot, \rho)$ is a continuous unitary representation of $U(k)$. We shall also equip $P(E, \rho)$ with the inner product 


$$
\left(F_{1} \mid F_{2}\right)=\sum_{j=1}^{d_{\rho}}\left(l^{j} \circ F_{1}, l^{j} \circ F_{2}\right) .
$$

Then, for all $y \in U(k)$

$$
\begin{aligned}
\left(D_{0}(y, \rho) F_{1} \mid D_{0}(y, \rho) F_{2}\right) & =\sum_{j=1}^{d_{\rho}}\left(l^{j} \circ D_{0}(y, \rho) F_{1}, l^{j} \circ D_{0}(y, \rho) F_{2}\right) \\
& =\sum_{j=1}^{d_{\rho}}\left(\widetilde{D}_{0}(y, \rho) l^{j} \circ F_{1}, \widetilde{D}_{0}(y, \rho) l^{j} \circ F_{2}\right) \\
& =\sum_{j=1}^{d_{\rho}}\left(l^{j} \circ F_{1}, l^{j} \circ F_{2}\right)=\left(F_{1} \mid F_{2}\right) \\
& =\sum_{j=1}^{d_{\rho}}\left(l^{j} \circ F_{1}, l^{j} \circ F_{2}\right)=\left(F_{1} \mid F_{2}\right) .
\end{aligned}
$$

Therefore, $D_{0}(\cdot, \rho)$ is continuous irreducible unitary representation of $U(k)$. By our choice of the basis $\left\{l^{1}, \ldots, l^{d} \rho\right\}$ we notice that $\Phi_{1}(P(E, \rho))=P(E, \xi)$ (see also Theorem 1.5). The following corollary may be deduced immediately from Theorem 3.8.

Corollary 3.10. The subspace $(P(E, \rho))^{\sim}$ of $S\left(E^{*}\right)$ may be decomposed into an orthogonal direct sum as

$$
(P(E, \rho))^{\sim}=\sum_{\alpha=1}^{d_{\rho}} \bigoplus(P(E, \rho))_{\alpha}^{\sim} .
$$

In addition, each $(P(E, \rho))_{\alpha}^{\sim}$ is (unitary) isomorphic to $P(E, \rho)$, and $(P(E, \rho))_{1}^{\sim}=P(E, \xi)$.

By an analogous reasoning, Corollary 3.2 and Theorem 3.8 lead us to

Corollary 3.11. The subspace $(H(E, \rho))^{\sim}$ of $H\left(E^{*}\right)$ may be decomposed into an orthogonal direct sum of simple $G_{0}$-modules as

$$
(H(E, \rho))^{\sim}=\sum_{\alpha=1}^{d_{\rho}} \bigoplus(H(E, \rho))_{\alpha}^{\sim}
$$

In addition, each $G_{0}$-module $(H(E, \rho))_{\alpha}^{\sim}$ is (unitary) isomorphic to the $G_{0^{-}}$ module $H(E, \rho)$, and $(H(E, \rho))_{1}^{\sim}=H(E, \xi)$.

Notice that a similar result to Corollary 3.10 was obtained by S. Gelbart [7],

The special class of $G$-harmonic polynomial functions in Corollary 3.7, was also studied in great detail by $H$. Maass [19]. Indeed, if we let $p_{0} \in S\left(E^{*}\right)$ be defined by $p_{0}(X)=\operatorname{det}\left(X X^{t}\right)$ for all $X \in E$, then $p_{0}(D)$ is precisely the differential operator introduced by $\mathrm{H}$. Maass [18]. Clearly, $p_{0} \in J_{+}\left(E^{*}\right)$, so that $H(E, m)$ 
is a subspace of all "harmonic forms" in [18]. These so-called "generalized spherical harmonic functions" lead to interesting questions in harmonic analysis. In the next section we shall investigate these "generalized spherical harmonic functions" for any $\xi\left(m_{1}, m_{2}, \ldots, m_{n}\right)$ with $m_{1} \geqslant m_{2} \geqslant \cdots \geqslant m_{n} \geqslant 0$.

4. Harmonic analysis on Stiefel manifolds. Suppose that $k>2 n$; then the Stiefel manifold $S^{n, k}$ consists of all matrices $s \in E_{0}$ such that $s s^{t}=1_{n}$, where $1_{n}$ denotes the identity matrix of order $n$. Let $H\left(S^{n, k}\right)$ denote the space of functions on $S^{n, k}$ obtained by restricting all $G$-harmonic polynomial functions on $E$ to the Stiefel manifold.

When $n=1$, the Stiefel manifold $S^{1, k}$ reduces to the $(k-1)$ unit sphere; the spherical harmonics are then obtained as restriction of the harmonic polynomials to the surface of this sphere. Using the spherical harmonics one can obtain a very interesting decomposition of $L^{2}\left(\mathbf{R}^{k}\right)$ into a direct sum of subspaces, invariant under the Fourier transform and under the action of the rotation group. The references to this classical subject are abundant; see for example Vilenkin [24, Chapter IX], Coifman and Weiss, [6, Chapter II] and Stein and Weiss, [21, Chapter IV]. The excellent treatment by Stein and Weiss seems to conform the most to our geometrical intuition.

This section is organized in the following way: The first part will be devoted to the establishment of the isomorphism between the $G$-harmonic polynomial functions and the Stiefel harmonics; and from this a decomposition of $L^{2}\left(S^{n, k}\right)$ will follow. The second part will treat the direct sum decomposition of $L^{2}\left(E_{0}\right)$ in connection with the Stiefel harmonics and the Fourier-Plancherel transform.

The results of the first part are original. The theorems in the second part may be regarded as a reformulation and unification of parts of the work of S. Gelbart [7] and C. Herz [13]. Nevertheless, the proofs involved in the second part are interesting, for it is somewhat surprising that the method given by Stein and Weiss [21] may be generalized word by word to our situation.

Recall that $\mathrm{I}=\left[1_{n}\right.$ 0] was defined in $\S 3$; clearly $\mathrm{I}$ belongs to $S^{n, k}$. If $s \in$ $S^{n, k}$, then the rows of $s$ can be extended to an orthonormal basis for $\mathbf{R}^{1 \times k}$ such that if $g$ is the matrix formed by $k$ orthogonal row vectors, then $g \in G_{0}$. Hence I $g=s$; from this we conclude that $G_{0}$ acts transitively on $S^{n, k}$. It follows that $S^{n, k}$ may be diffeomorphically identified with the homogeneous space $S O(k-n) \backslash S O(k)$. In this section, we shall prove that the restriction mapping $f \rightarrow$ $f / S^{n, k}\left(f \in H\left(E^{*}\right)\right)$ is an isomorphism. For this purpose, we first need

Proposition 4.1. Let $E$ be a finite dimensional vector space over the complex field. Assume that $G \subset \operatorname{Aut}(E)$ is a connected linear algebraic group such that $G$ is the complexification of a connected subgroup $G_{0}$ of the automorphism group $\operatorname{Aut}(E)$ of $E$. Suppose that the mapping $g \rightarrow X g$ from $G$ to $E$ is holomor- 
phic for a fixed $X \in E$ (here $X g$ denotes the action of $G$ on $X$ ).

Let $f$ be a holomorphic function on $E$. Then the following statements are equivalent:

(i) $f(X g)=0$ for all $g \in G_{0}$,

(ii) $f(X g)=0$ for all $g \in G$.

Proof. The proof of elementary. For details see [23].

Let $S\left(S^{n, k}\right)$ be the ring of all functions on $S^{n, k}$ obtained by restricting elements of $S\left(E^{*}\right)$ to $S^{n, k}$. Then we have

THEOREM 4.2. The restriction mapping $f \rightarrow f / S^{n, k}\left(f \in H\left(E^{*}\right)\right)$ is a $G_{0}$-module isomorphism of $H\left(E^{*}\right)$ onto $S\left(S^{n, k}\right)$.

Proof. Let $\theta$ denote this restriction mapping. Then it is clear that $\theta$ is linear. By Theorem 2.5, $S\left(E^{*}\right)=J\left(E^{*}\right) \otimes H\left(E^{*}\right)$; since $J\left(E^{*}\right)$ reduces to scalars on the Stiefel manifold, it follows that $\theta$ is surjective and that $S\left(S^{n, k}\right)=H\left(S^{n, k}\right)$. Let us show that $\theta$ is injective. First, observe that $I$ is quasi-regular, i.e., that $P \subset$ $\overline{U_{c \in C} * O_{c I}}$. This can be proved as follows:

We equip $E$ with the norm $\|\cdot\|$ defined by $\|X\|=\operatorname{tr}\left(X \bar{X}^{t}\right)^{1 / 2}$, and recall that $Z_{0}=\left[\left(I_{n}\right)(0)\left(-I_{n}^{\prime}\right)(0)\right]$, as defined in $\S 3$, is such that $O_{Z_{0}}=P_{n}=$ $\left\{X \in E: X X^{t}=0, \operatorname{rank}(X)=n\right\}$. Now for any $\epsilon>0$, let $c=c(\epsilon)$ be a fixed square root of $2 \epsilon(1-i+\epsilon)$. Let $g \in \operatorname{GL}(k, \mathrm{C})$ be given in block form as $\left[\begin{array}{cc}D & D^{\prime} \\ -D^{\prime} & D\end{array}\right]$ (we suppose as usual that $k$ is even; the case $k$ odd may be treated in a similar fashion); here $D$ (resp. $D^{\prime}$ ) is a diagonal matrix of order $k / 2$ with diagonal entries all equal to $(1+\epsilon) / c$ (resp. $(-i+\epsilon) / c)$. Now, by a simple computation one can see that $g \in G$ and

$$
\left\|c(\epsilon) \mathrm{I} g-Z_{0}\right\|=(2 n)^{1 / 2} \epsilon .
$$

Any element of $O_{Z_{0}}$ is of the form $Z_{0} g_{1}$ for some $g_{1} \in G$. Since the mapping $X \rightarrow X g_{1}$ from $E$ to $E$ is continuous, equation (4.1) implies that any element of $O_{Z_{0}}$ may be obtained as limit of elements in $U_{c \in \mathrm{C}^{*}} \mathrm{O}_{c \mathrm{I}}$.

Now Proposition 5 (p. 343) of [15] implies that the mapping $\gamma_{\mathrm{I}}: H\left(E^{*}\right) \rightarrow$ $S\left(O_{\mathrm{I}}\right)$ is a $G$-module isomorphism (see [15] for definitions and notations). Now if $f \in H\left(E^{*}\right)$ and $\theta f=0$, then since $S^{n, k}=\mathrm{I} G_{0}$, we have $f(\mathrm{I} g)=0$ for all $g \in$ $G_{0}$. From Proposition 4.1 it follows that $f(\mathrm{I} g)=0$ for all $g \in G$. But this just means that $\gamma_{\mathrm{I}}(f)=0$. Therefore $f=0$ and, hence, $\theta$ is injective. Now it is clear that $\theta$ is a $G_{0}$-module isomorphism.

Recall that the representation $R_{0}(\cdot, \xi)=R(\cdot, \xi) / G_{0}$ of $G_{0}$ on $H(E, \xi)$ is irreducible. Let $H\left(S^{n, k}, \xi\right)$ denote the subspace of $H\left(S^{n, k}\right)$ which is obtained by restricting the $G$-harmonic $\xi$-covariant polynomials to the Stiefel manifold $S^{n, k}$. Let $R_{0}^{\#}(\cdot, \xi)$ be the representation of $G_{0}$ on $H\left(S^{n, k}, \xi\right)$ obtained by right translation; then Theorem 4.2 immediately implies the following result. 
CoROllary 4.3. The restriction mapping $f \rightarrow f / S^{n, k} \quad(f \in H(E, \xi))$ is an isomorphism intertwining $R_{0}(\cdot, \xi)$ and $R_{0}^{\#}(\cdot, \xi)$ : hence, $R_{0}^{\#}(\cdot, \xi)$ is irreducible with highest weight

$$
\underbrace{\zeta\left(m_{1}, m_{2}, \ldots, m_{n}, 0 \cdots 0\right)}_{[k / 2]} .
$$

REMARKS 4.4. (i) With regard to the proof of Theorem 4.2 it is interesting to note that $O_{1}=\left\{X \in E: X X^{t}=1_{n}\right\}$. Indeed, to show this we remember from Lemma 2.8 that the symmetric bilinear form $(\cdot \mid \cdot)$ was defined on $\mathbf{C}^{1 \times k}$ by $(x \mid y)=x y^{t}$. If $X \in E$ and $X X^{t}=1_{n}$, then the $n$ rows of $X$, say $x_{1}, x_{2}$, $\ldots, x_{n}$, constitute an orthonormal system for $\mathbf{C}^{1 \times k}$. Let $V=V_{X}$ be the subspace (of $\mathrm{C}^{1 \times k}$ ) spanned by $x_{1}, \ldots, x_{n}$. Now the restriction of $(\cdot \mid \cdot)$ to $V$ is nondegenerate. For if $x \in V$, then $x=\Sigma_{i=1}^{n} c_{i} x_{i}$, and $\left(x \mid x_{i}\right)=c_{i}$. It follows that $\mathbf{C}^{1 \times k}=V \oplus V^{\perp}$. Also, $(\cdot \mid \cdot)$ restricted to $V$ is nondegenerate, because if $y \in V^{\perp}$ is such that $(y \mid z)=0$ for all $z \in V^{\perp}$, then $y \in\left(V^{\perp}\right)^{\perp}=V$, hence, $y \in$ $V^{\perp} \cap V$ and $y=0$. Therefore, $V^{\perp}$ possesses an orthonormal basis with respect to $(\cdot 1 \cdot)$, say $x_{n+1}, \ldots, x_{k}$. Clearly, the system $\left\{x_{1}, x_{2}, \ldots, x_{k}\right\}$ is an orthonormal basis (with respect to the bilinear form $(\cdot \mid \cdot)$ of $\mathbf{C}^{1 \times k}$ ). Let $g$ be the $k$ by $k$ matrix with columns $x_{1}^{t}, x_{2}^{t}, \ldots, x_{k}^{t}$. By replacing, if necessary, $x_{n+1}$ by $-x_{n+1}$ we may assume that $g \in G$. Next, it is clear that $X g=\mathbf{I}$, and from this our desired conclusion follows immediately.

(ii) A more direct proof may be given for Corollary 4.3. Indeed, let us denote by $H\left(E_{0}, \xi\right)$ the linear space of all complex-valued polynomial functions on $E_{0}$ which is obtained by restricting elements of $H(E, \xi)$ to $E_{0}$. By analytic continuation the restriction mapping $f \rightarrow f / E_{0} \quad\left(f \in H\left(E^{*}\right)\right)$ is an isomorphism. Next, set $B_{0}=B \cap \mathrm{GL}(n, \mathbf{R})$, and let $H\left(E_{0}^{*}\right)$ denote the linear space consisting of all $G_{0}$-harmonic, complex-valued polynomial functions on $E_{0}$. Then it follows that $H\left(E_{0}, \xi\right)$ is a subspace of $H\left(E_{0}^{*}\right)$ such that if $f \in H\left(E_{0}, \xi\right)$, then $f(b X)=$ $\xi(b) f(X)$ for all $(b, X) \in B_{0} \times E_{0}$. Moreover, the representation of $G_{0}$ obtained by right translation on $H\left(E_{0}, \xi\right)$ is irreducible. Now by an argument using the Gram-Schmidt process, similar to the one used to obtain the Iwasawa decomposition for $\mathrm{GL}(n, \mathbf{R})$ (see, e.g., [14, Theorem 14, p. 305]), we may conclude that $E_{0}$ is essentially $B_{0} S^{n, k}$. From this fact we infer that the restriction mapping $f \rightarrow f / S^{n, k}\left(f \in H\left(E_{0}, \xi\right)\right)$ is a $G_{0}$-module isomorphism. Hence, $R_{0}^{\#}(\cdot, \xi)$ is irreducible.

Now set $K_{0}=S O(k-n)$ and let $d s$ denote the unique normalized, regular, $G_{0}$-invariant Baire measure on the homogeneous space $K_{0} \backslash G_{0}$. Let $R_{0}^{\#}$ denote the continuous unitary representation of $G_{0}$ which is obtained by right translation on $L^{2}\left(K_{0} \backslash G_{0}\right)$, the Hilbert space of square integrable complex-valued functions on $K_{0} \backslash G_{0}$. In addition, we shall designate by $\hat{G}_{0}$ a set of representatives of 
equivalence classes of continuous irreducible unitary representations of $G_{0}$. By the Peter-Weyl theory, we then have the following decomposition of $L^{2}\left(K_{0} \backslash G_{0}\right)$ into an orthogonal direct sum of $R_{0}^{\#}$-invariant subspaces:

$$
L^{2}\left(K_{0} \backslash G_{0}\right)=\sum_{\lambda \in \hat{G}_{0}} \bigoplus P_{\lambda} L^{2}\left(K_{0} \backslash G_{0}\right),
$$

where $P_{\lambda}=d_{\lambda} \int_{G_{0}} \operatorname{tr} \bar{\lambda}(g) R_{0}^{\#}(g) d g$ and $d_{\lambda}$ is the degree of $\lambda$. In general, the restriction of $R_{0}^{\#}$ to $P_{\lambda} L^{2}\left(K_{0} \backslash G_{0}\right)$ is not irreducible but "primary", i.e.,

$$
P_{\lambda} L^{2}\left(K_{0} \backslash G_{0}\right)=\sum_{\mu} \bigoplus V_{\mu}^{\lambda}
$$

with $R_{0}^{\#} / V_{\mu}^{\lambda}$ unitary equivalent to $\lambda$ for all $\mu$. A continuous irreducible unitary representation $\lambda$ of $G_{0}$ on a Hilbert space $H_{\lambda}$ is said to be of class 1 with respect to $K_{0}$ if there exists a nonzero vector $h_{0} \in H_{\lambda}$ such that $\lambda(k) h_{0}=h_{0}$ for every $k \in K_{0}$ (cf. [11, p. 414] for details). Set

$$
W_{\lambda}=\left\{h \in H_{\lambda}: \lambda(k) h=h, \forall k \in K_{0}\right\} .
$$

Then clearly $W_{\lambda}$ is a subspace of $H_{\lambda}$. Under the above assumptions, the following classical result is valid. A similar result, valid in a more general context, has been proved by Gross and Kunze [8, Theorem 2, p. 129].

Proposition 4.5. The projection $P_{\lambda}$ is of finite rank, and $P_{\lambda}$ is nonzero if and only if $\lambda$ is of class 1 with respect to $K_{0}$. In addition, the number of times that $\lambda$ occurs in the reduction of $R_{0}^{\#}$ is precisely the dimension $m_{\lambda}$ of the space $W_{\lambda}$.

PRoof. We sketch the proof. First, observe that $m_{\lambda}=\operatorname{dim} W_{\lambda}$ is equal to the multiplicity of the trivial representation $1_{K_{0}}$ in the reduction of $\lambda / K_{0}$, the restriction of $\lambda$ to $K_{0}$. Next, by the Frobenius reciprocity theorem, the number of times that $1_{K_{0}}$ occurs in the reduction of $\lambda / K_{0}$ is equal to the number of times that $\lambda$ occurs in the reduction of the induced representation ind $K_{0} \uparrow G_{0} 1_{K_{0}}$. But the latter is merely the representation $R_{0}^{\#}$ on $L^{2}\left(K_{0} \backslash G_{0}\right)$. In conclusion, the above facts together with equation (4.3) imply that

$$
\operatorname{dim} P_{\lambda} L^{2}\left(K_{0} \backslash G_{0}\right)=m_{\lambda} \operatorname{dim} V_{\lambda}^{\mu}=\operatorname{dim} W_{\lambda} \operatorname{dim} V_{\lambda}^{\mu}<\infty .
$$

Finally, it is clear that $\lambda$ is of class 1 with respect to $K_{0}$ if and only if $\operatorname{dim} W_{\lambda}>$ 0 . The last part of the proposition now follows immediately from equation (4.4).

We shall say that $P_{\lambda} L^{2}\left(K_{0} \backslash G_{0}\right)$ is the subspace of $L^{2}\left(K_{0} \backslash G_{0}\right)$ which transforms under $R_{0}^{\#}$ according to $\lambda$. By Theorem 1.1, we also know that any $\lambda \in \hat{G}_{0}$ may be obtained by the inducing process of Theorem 1.1. Therefore, we may set $\lambda=\pi_{0}(\cdot, \zeta)$; where $\zeta=\zeta\left(m_{1}, m_{2}, \ldots, m_{[k / 2]}\right)$ is a dominant character of the maximal torus $T$ of $G_{0}$ (see Lemma 3.3). The integers $m_{i}$ in the dominant character $\zeta$ must satisfy the following conditions: 


$$
m_{1} \geqslant m_{2} \geqslant \cdots \geqslant m_{[k / 2]} \geqslant 0 \text { when } k \text { is odd, }
$$

and

$$
m_{1} \geqslant m_{2} \geqslant \cdots \geqslant\left|m_{k / 2}\right| \text { when } k \text { is even }
$$

(cf. [28, p. 41] for details).

Now by Proposition 2.1 (p. 32) of [7] a representation $\pi_{0}(\cdot, \zeta)$ is of class 1 with respect to $K_{0}$ if and only if $\zeta$ is of the form

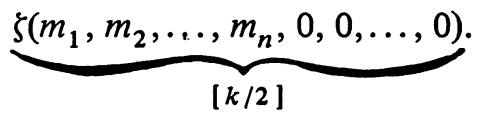

Therefore, we may rewrite equation (4.2) as

$$
L^{2}\left(K_{0} \backslash G_{0}\right)=\sum_{\zeta} \bigoplus P_{\pi_{0}(\cdot, \zeta)}\left(L^{2}\left(K_{0} \backslash G_{0}\right)\right)
$$

where the summation ranges over all $\zeta \in \hat{T}$ with

$$
\zeta=\underbrace{\zeta\left(m_{1}, m_{2}, \ldots, m_{n}, 0, \ldots, 0\right)}_{[k / 2]}
$$

and $m_{1} \geqslant m_{2} \geqslant \cdots \geqslant m_{n} \geqslant 0$. Now recall that $K_{0} \backslash G_{0}$ may be identified with the Stiefel manifold; thus the above results together with Corollary 3.11 of $\S 3$ will enable us to obtain a concrete decomposition of $L^{2}\left(S^{n, k}\right)$.

Let $(\cdot, \cdot)_{S}$ denote the inner product defined on $L^{2}\left(S^{n, k}\right)$ by

$$
(f, h)_{S}=\int_{S^{n, k}} f(s) \overline{h(s)} d s .
$$

Recall that $K_{0}$ is the stability subgroup of $I=\left[1_{n} 0\right]$, so that $G_{0}$ acts transitively on $S^{n, k}$. Next, for every continuous complex-valued function $f$ on $S^{n, k}$ we have

$$
\int_{S^{n, k}} f(s) d s=\int_{G_{0}} f(\mathbf{I} g) d g .
$$

Indeed, to establish (4.6) we observe that $d s$ is $G_{0}$-invariant so that

$$
\int_{S^{n, k}} f(s) d s=\int_{S^{n, k}} f(s g) d s \quad \text { for all } g \in G_{0} .
$$

And by Fubini's theorem

$$
\begin{aligned}
\int_{s^{n, k}} f(s g) d s & =\int_{G_{0}}\left\{\int_{s^{n, k}} f(s g) d s\right\} d g \\
& =\int_{S^{n, k}}\left\{\int_{G_{0}} f(s g) d g\right\} d s .
\end{aligned}
$$

For every $s \in S^{n, k}$ there exists $g_{s} \in G_{0}$ such that $s=I g_{s}$. Therefore, by performing the change of variable $g \rightarrow g_{s}^{-1} g$ in the inner integral of the last equation, 
and by observing that $G_{0}$ is unimodular, we get

$$
\int_{S^{n, k}}\left\{\int_{G_{0}} f(s g) d g\right\} d s=\int_{S^{n, k}}\left\{\int_{G_{0}} f(\mathrm{I} g) d g\right\} d s=\int_{G_{0}} f(\mathbf{I} g) d g .
$$

Next, set

$$
\left(H\left(S^{n, k}, \rho\right)\right)^{\sim}=\left\{f / S^{n, k}: f \in(H(E, \rho))^{\sim}\right\}
$$

where the subspace $(H(E, \rho))^{\sim}$ of $H\left(E^{*}\right)$ is the space defined in $\S 3$ (cf. Corollary 3.11). By Theorem 4.2, as a $G_{0}$-module $\left(H\left(S^{n, k}, \rho\right)\right)^{\sim}$ is isomorphic to the $G_{0}$ module $(H(E, \rho))^{\sim}$. Now by the proof of Theorem 3.1 and, in particular, by Lemma 3.5, it follows that $\left(H\left(S^{n, k}, \rho\right)\right)^{\sim}$ consists of functions which transform according to $\pi_{0}(\cdot, \zeta)$ (recall that $\rho=\pi(\cdot, \xi)$ is a representation of $\operatorname{GL}(n, \mathbf{R})$ and that in Lemma $3.5 \pi_{0}(\cdot, \zeta)$ was obtained by applying the inducing process of Theorem 1.1 to

$$
\underbrace{\zeta=\zeta\left(m_{1}, m_{2}, \ldots, m_{n}, 0, \ldots, 0\right)}_{[k / 2]}
$$

where the indices $m_{i}$ are those in $\xi\left(m_{1}, m_{2}, \ldots, m_{n}\right)$. We also have the following decomposition of $\left(H\left(S^{n, k}, \rho\right)\right)^{\sim}$ into an orthogonal direct sum

$$
\left(H\left(S^{n, k}, \rho\right)\right)^{\sim}=\sum_{\alpha=1}^{d_{\rho}} \bigoplus\left(H\left(S^{n, k}, \rho\right)\right)_{\alpha}^{\sim}
$$

In equation (4.7) the restriction of $R_{0}^{\#}$ to each $\left(H\left(S^{n, k}, \rho\right)\right)_{\alpha}^{\sim}$ is unitary equivalent to $\pi_{0}(\cdot, \zeta)$. Now according to Proposition 2.2 in [7] (referred to as an "Act of Providence", p. 33), the number of times that $\pi_{0}(\cdot, \zeta)$ occurs in the reduction of $R_{0}^{\#}$ is exactly the degree $d_{\rho}$ of the representation $\rho$. The argument for this involves repeated applications of the branching theorem. A more general result with a more straightforward proof will appear in [9]. From this last fact and equation (4.7), we infer that $\left(H\left(S^{n, k}, \rho\right)\right)^{\sim}$ is precisely the subspace of $L^{2}\left(S^{n, k}\right)$ which transforms according to $\pi_{0}(\cdot, \zeta)$. To unify the notations we set

$$
H\left(S^{n, k}\right)_{\pi_{0}(\cdot, \zeta)}=\left(H\left(S^{n, k}, \rho\right)\right)^{\sim}, \quad H\left(S^{n, k}\right)_{\pi_{0}(\cdot, \zeta)}^{\alpha}=\left(H\left(S^{n, k}, \rho\right)\right)_{\alpha}^{\sim} .
$$

Similarly, we set

$$
H\left(E_{0}\right)_{\pi_{0}(\cdot, \zeta)}=\left(H\left(E_{0}, \rho\right)\right)^{\sim}, \quad H\left(E_{0}\right)_{\pi_{0}(: \zeta)}^{\alpha}=\left(H\left(E_{0}, \rho\right)\right)_{\alpha}^{\sim} .
$$

Now let $\pi_{0}\left(\cdot, \zeta_{1}\right)$ and $\pi_{0}\left(\cdot, \zeta_{2}\right)$ be two different class 1 representations of $G_{0}$; we shall show that $H\left(S^{n, k}\right)_{\pi_{0}}\left(\cdot, \zeta_{1}\right)$ and $H\left(S^{n, k}\right)_{\pi_{0}\left(\cdot, \zeta_{2}\right)}$ are orthogonal. For this we observe that it is sufficient to show the following: If $\left\{f_{1}^{\alpha}, f_{2}^{\alpha}, \ldots, f_{s}^{\alpha}\right\}$ is an orthonormal basis for $H\left(S^{n, k}\right)_{\pi_{0}}^{\alpha}\left(\cdot, \zeta_{1}\right)$ and $\left\{f_{1}^{\beta}, f_{2}^{\beta}, \ldots, f_{t}^{\beta}\right\}$ is an orthonormal basis for $H\left(S^{n, k}\right)_{\pi_{0}}^{\beta}\left({ }^{\circ}, \xi_{2}\right)$ then $\left(f_{i}^{\alpha}, f_{j}^{\beta}\right)_{S}=0$ for all $i, j, 1 \leqslant i$ $\leqslant s, 1 \leqslant j \leqslant t$. Indeed, 


$$
\begin{aligned}
\left(f_{i}^{\alpha}, f_{j}^{\beta}\right)_{S} & =\int_{S^{n, k}} f_{i}^{\alpha}(s) \overline{f_{j}^{\beta}(s)} d s \\
& =\int_{G_{0}} f_{i}^{\alpha}(\mathrm{I} g) \overline{f_{j}^{\beta}(\mathrm{I} g)} d g \quad \text { by }(4.6) \\
& =\int_{G_{0}}\left(R_{0}^{\#}(g) f_{i}^{\alpha}\right)(\mathrm{I})\left(R_{0}^{\#}(g) \overline{f_{j}^{\beta}}\right)(\mathrm{I}) d g .
\end{aligned}
$$

Because the restrictions of the representation $R_{0}^{\#}$ to $H\left(S^{n, k}\right)_{\pi_{0}}^{\alpha}\left(\cdot, \zeta_{1}\right)$ and to $H\left(S^{n, k}\right)_{\pi_{0}}^{\beta}\left(\cdot, \xi_{2}\right)$, respectively, belong to different classes of irreducible unitary representations of $G_{0}$, the orthogonality relations immediately imply that the last integral in the above equation is equal to zero.

We summarize the above results in a more concise fashion in

THEOREM 4.6. The Hilbert space $L^{2}\left(S^{n, k}\right)$ decomposes into an orthogonal direct sum of its subspaces as follows:

$$
L^{2}\left(S^{n, k}\right)=\sum_{\zeta} \bigoplus H\left(S^{n, k}\right)_{\pi_{0}}(\cdot, \zeta),
$$

where each subspace $H\left(S^{n, k}\right)_{\pi_{0}(\cdot, \zeta)}$ transforms according to the irreducible unitary representation $\pi_{0}(\cdot, \zeta)$ of $G_{0}$, and where the summation ranges over all representations $\pi_{0}(\cdot, \zeta)$ which are induced from the dominant weights

$$
\zeta=\underbrace{\zeta\left(m_{1}, m_{2}, \ldots, m_{n}, 0, \ldots, 0\right)}_{[k / 2]}
$$

of $G_{0}$. In addition, each subspace $H\left(S^{n, k}\right)_{\pi_{0}}(\cdot, \zeta)$, which consists of Stiefel harmonic functions, is invariant under the representation $R_{0}^{\#}$ of $G_{0}$ on $L^{2}\left(S^{n, k}\right)$.

Notes 4.7. (i) When $n=1$ and $k>2$, Theorem 4.6 reduces to the classical decomposition of the Hilbert space $L^{2}\left(S^{k-1}\right)$ into orthodirect sum of homogeneous spherical harmonic subspaces (see, e.g., [21, p. 141]).

(ii) In the decomposition (4.10) when $\zeta=\zeta(m, m, \ldots, 0, \ldots, 0)$, it is easy to see that $\operatorname{deg} \rho=1$ (cf. [26, Theorem 7.5B, p. 201]), so that $H\left(S^{n, k}\right)_{\pi_{0}}(\cdot, \zeta)$ is then reduced to the subspace of all determinantly homogeneous Stiefel harmonics of degree $m$ (cf. Corollary 3.7 of $\S 3$ ).

Recall that for every $\alpha$, the linear space $H(E)_{\pi_{0}}^{\alpha}(\cdot, \zeta)$ was equipped with the inner product $(\cdot, \cdot)$ defined by $(p, q)=(p(D) \tilde{q})(0)$. Now if we denote by $R_{0}^{\alpha}(\cdot, \zeta)$ the representation of $G_{0}$ obtained by right translation on $H(E)_{\pi_{0}}^{\alpha}(\cdot, \zeta)$, then by Corollary $3.11, R_{0}^{\alpha}(\cdot, \zeta)$ is irreducible. Since the $G$-harmonic polynomials are determined by their restrictions to the Stiefel manifold $S^{n, k}$, we may equip $H(E)_{\pi_{0}}^{\alpha}(\cdot, \zeta)$ with the inner product $(\cdot, \cdot)_{S}$ defined by

$$
(p, q)_{S}=\int_{S^{n, k}} p(s) \overline{q(s)} d s \quad \forall p, q \in H(E)_{\pi_{0}}^{\alpha}(\cdot, \zeta) .
$$


Proposition 4.8. There exists a constant $C=C\left(\alpha, \pi_{0}(\cdot, \zeta)\right)$ such that

$$
(p, q)_{S}=C(p, q) \quad \forall p, q \in H(E)_{\pi_{0}}^{\alpha}(\cdot, \zeta) \cdot
$$

Proof. The proof is elementary.

The next part will be devoted to the study of $L^{2}\left(E_{0}\right)$, the Hilbert space of square-integrable functions on $E_{0}$. For this purpose, we let $P_{n}$ denote the cone of positive definite matrices of order $n$. Then we may write almost all $X \in E_{0}$ uniquely in the form $X=r^{1 / 2} s=r[X] s[X]$ where $r \in P_{n}$ and $s \in S^{n, k}$. Corresponding to this decomposition of $E_{0}$ we have the volume element $d X=$ $\left(1 / 2^{n}\right)|r|^{1 / 2(k-n-1)} d s d r$, where $d s$ is the unique measure on $S^{n, k}$ invariant under the action of $G_{0}$ (cf. [13, p. 482]).

A function $f$ on $E_{0}$ is said to be radial if it depends only on its "radial part", i.e., $f(X)=f \sqrt{ }(r[X])$ for some function $f \sqrt{ }$ defined on $P_{n}$. It is easily seen that this is equivalent to the statement that $f$ is $G_{0}$-invariant. For every $h \sqrt{ } \in$ $H\left(S^{n, k}\right)_{\pi_{0}(\cdot, \zeta)}$ we define a function $h$ on $E_{0}$ by setting $h(X)=h^{\sqrt{ }}(s[X])$ where $s[X]$ is the "Stiefel part" of $X$ (of course, $h$ is defined only almost everywhere on $\left.E_{0}\right)$. Now let $H\left(E_{0}\right)_{\pi_{0}}(\cdot, \zeta)$ denote the subspace of $L^{2}\left(E_{0}\right)$ which is spanned

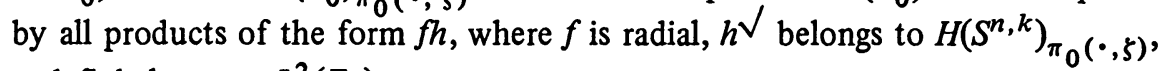
and $f h$ belongs to $L^{2}\left(E_{0}\right)$.

From Theorem 4.6 we deduce the following result.

Corollary 4.9. The Hilbert space $L^{2}\left(E_{0}\right)$ may be decomposed into an orthogonal direct sum of the form

$$
L^{2}\left(E_{0}\right)=\sum_{\zeta} \bigoplus H\left(E_{0}\right)_{\pi_{0}}(\cdot, \zeta)
$$

where the sum ranges over all dominant weights $\zeta\left(m_{1}, m_{2}, \ldots, m_{n}, 0, \ldots, 0\right)$ (of $G_{0}$ ) with $m_{1} \geqslant m_{2} \geqslant \cdots \geqslant m_{n} \geqslant 0$.

Proof. We sketch the proof. The fact that every $H\left(S^{n, k}\right)_{\pi_{0}}(\cdot, \zeta)$ is finite dimensional implies readily that every $H\left(E_{0}\right)_{\pi_{0}(\cdot, \zeta)}$ is a closed subspace of $L^{2}\left(E_{0}\right)$. The mutual orthogonality of the $H\left(E_{0}\right)_{\pi_{0}}(\cdot, \zeta)$ follows immediately from the mutual orthogonality of the $H\left(S^{n, k}\right)_{\pi_{0}(\cdot, \xi)}$. The result now follows if we observe that $L^{2}\left(E_{0}\right)=L^{2}\left(P_{n}\right) \otimes L^{2}\left(S^{n, k}\right)$ and that $L^{2}\left(S^{n, k}\right)=$ $\Sigma_{\zeta} \oplus H\left(S^{n, k}\right)_{\pi_{0}(\cdot, \zeta)}$.

In the classical case when $n=1$, the decomposition (4.11) possesses the additional property that the Fourier transform maps each summand onto itself. In [21], this fact was obtained from the crucial observation that the subspace of each linear space $H^{j}\left(E_{0}^{*}\right)$ which is invariant under the action of $K_{0}$ (the stability subgroup of the pole $I=(1,0, \ldots, 0))$ is of dimension 1 . This optimal situation occurs also in our case if we consider the subspaces $H\left(E_{0}\right)_{\pi_{0}}(\cdot, \zeta)$ with $\zeta=$ $\zeta(m, 0,0, \ldots, 0)$ or 


$$
\underbrace{\zeta=\zeta(m, m, \ldots, m, 0, \ldots, 0)}_{[k / 2]}
$$

It is easy to see that with $\zeta=\zeta(m, 0, \ldots, 0)$ we obtain again the classical case $(n=1)$; on the other hand, when $\zeta=\zeta(m, \ldots, m, 0, \ldots, 0), H\left(S^{n, k}\right)_{\pi_{0}(\cdot, \zeta)}$ is reduced to the subspace of all determinantly homogeneous Stiefel harmonics of degree $m$. In the remainder of this section, we shall investigate this situation.

For $f$ in $L^{1}\left(E_{0}\right) \cap L^{2}\left(E_{0}\right)$, we define the Fourier transform of $f$ (denoted Ff) by setting

$$
F f(X)=\int_{E_{0}} \exp (-2 \pi i(X \mid Y)) f(Y) d Y ; \quad(X \mid Y)=\operatorname{tr}\left(X Y^{t}\right)
$$

Recall that the representation of $G_{0}$ obtained by right translation on $H\left(E_{0}, m\right)$ was denoted by $R_{0}(\cdot, m)$. To this corresponds the representation $R_{0}^{\#}(\cdot, m)$ on $H\left(S^{n, k}, m\right)$.

Let $s \in S^{n, k}$, then the mapping $f \rightarrow f(s)\left(f \in H\left(E_{0}, m\right)\right)$ is a nonzero linear functional on $H\left(E_{0}, m\right)$. Therefore there exists a unique function $Z_{s}^{m} \in$ $H\left(E_{0}, m\right)$ such that, $f(s)=\left(f, Z_{s}^{m}\right)_{s}$ for every $f$ in $H\left(E_{0}, m\right)$.

Definition 4.10. The function $Z_{s}^{m}$ will be called the zonal harmonic function of $H\left(E_{0}, m\right)$ with pole $s$. The zonal harmonic function with pole $\mathbf{I}$ is particularly interesting and will subsequently play an important role. To simplify the situation, we remark that a function $f \in H\left(E_{0}, m\right)$ is $G_{0}$-harmonic if and only if $f$ is harmonic, i.e. $\Sigma_{i=1}^{n} \Delta_{i i} f=0$ (cf. [13, p. 484]). In [7], Gelbart proved that every polynomial on $E_{0}$ of $\rho$-covariant type is $G_{0}$-harmonic if and only if $f$ is harmonic. We shall obtain a similar result for the $\xi$-covariant polynomial functions on $E$.

Proposition 4.11. Let $f$ be any element of $H(E, \xi)$; then $f$ is $G$-harmonic if and only if $f$ is harmonic.

Proof. It is obvious that if $f$ is $G$-harmonic then $f$ is harmonic. Now assume that $\Sigma_{i=1}^{n} \Delta_{i i} f=0$. We shall show that $\Delta_{i j} f=0$ for all $i, j, 1 \leqslant i \leqslant j \leqslant n$. Recall that in the proof of Corollary 3.2 of $\S 3$, we defined the linear map $L(a)$ by $L(a) X=a X, X \in E, a \in \mathrm{GL}(n, \mathrm{C})$. Also, equation (3.14) implies that

$$
\left[\Delta_{i i}(f \circ L(b))\right](X)=\sum_{r, s=1}^{n} b_{r i} b_{s i}\left[\Delta_{r s} f\right](Y)
$$

$$
\text { for all } b \in B, X=E \text { and } Y=b X \text {. }
$$

Now the fact that $f$ is $\xi$-covariant implies that $\xi(b) f=f \circ L(b)$ and

$$
\sum_{i=1}^{n}\left[\Delta_{i i}(f \circ L(b))\right](X)=\xi(b) \sum_{i=1}^{n}\left[\Delta_{i i} f\right](X)=0
$$


Now let $b \in B$ with $b_{i i} \neq 0, i=1, \ldots, n, b_{t j} \neq 0$ for some fixed $t$ and $j(t>j)$, and $b_{r s}=0$ otherwise. Then, for such a $b$, equation (4.11) implies that

$$
\begin{aligned}
& {\left[\Delta_{i i}(f \circ L(b))\right](X)=b_{i i}^{2}\left[\Delta_{i i} f\right](Y) \quad \text { if } i \neq j,} \\
& {\left[\Delta_{j j}(f \circ L(b))\right](X)} \\
& \quad=b_{i j}^{2}\left[\Delta_{i j} f\right](Y)+2 b_{t j} b_{j j}\left[\Delta_{t j} f\right](Y)+b_{t j}^{2}\left[\Delta_{t t} f\right](Y) .
\end{aligned}
$$

Now it is easy to see from equations (4.13) and (4.14) that

$$
\begin{array}{r}
\sum_{i=1}^{n} b_{i i}^{2}\left[\Delta_{i i} f\right](X)+2 b_{t j} b_{i j}\left[\Delta_{t j} f\right](X)+b_{t j}^{2}\left[\Delta_{t t} f\right](X)=0 \\
\text { for every } X \in E .
\end{array}
$$

Equation (4.15) represents a homogeneous system of linear equations with unknowns $\left[\Delta_{i i} f\right](X), 1 \leqslant i \leqslant n$, and $\left[\Delta_{t j} f\right](X)$. From the arbitrariness of the coefficients $b_{i i}$ and $b_{t i}$, we infer that $\left[\Delta_{t i} f\right](X)=0$ and $\left[\Delta_{i i} f\right](X)=0$ for all $i=1, \ldots, n$ and all $X \in E$. From this it follows immediately that $f$ is $G$-harmonic.

Now let $d_{m}$ denote the degree of $R_{0}(\cdot, m)$. Then the following result is true. then

LEMMA 4.12. (a) If $\left\{f_{1}, \ldots, f_{d_{m}}\right\}$ is an orthonormal basis for $H\left(E_{0}, m\right)$

$$
Z_{s_{1}}^{m}\left(s_{2}\right)=\sum_{i=1}^{d_{m}} \overline{f_{i}\left(s_{1}\right)} f_{i}\left(s_{2}\right) .
$$

(b) $Z_{s}^{m}$ is real-valued and $Z_{s_{1}}^{m}\left(s_{2}\right)=Z_{s_{2}}^{m}\left(s_{1}\right)$ for all $s, s_{1}, s_{2} \in S^{n, k}$.

(c) For all $g \in G_{0}, R_{0}(g, m) Z_{s g}^{m}=Z_{s}^{m}$. must have

Proof. (a) Since $\left\{f_{1}, \ldots, f_{d_{m}}\right\}$ is an orthonormal basis for $H\left(E_{0}, m\right)$ we

$$
Z_{s_{1}}^{m}=\sum_{i=1}^{d_{m}}\left(Z_{s_{1}}^{m}, f_{i}\right)_{S} f_{i}=\sum_{i=1}^{d_{m}} \overline{f_{i}\left(s_{1}\right)} f_{i},
$$

so that $Z_{s_{1}}^{m}\left(s_{2}\right)=\Sigma_{i=1}^{d_{m}} \overline{f_{i}\left(s_{1}\right)} f_{i}\left(s_{2}\right)$.

(b) To prove that $Z_{s}^{m}$ is real valued we observe that we can choose the orthonormal basis in (a) to consist of real valued functions. Indeed, set $\Delta=$ $\sum_{i=1}^{n} \Delta_{i i}$ and observe that $\Delta f=0$ if and only if $\Delta \bar{f}=0$. Therefore the system $\left\{\operatorname{Re}\left(f_{i}\right), \operatorname{Im}\left(f_{i}\right)\right\}_{i}$ is a set of generators for $H\left(E_{0}, m\right)$. Choose from this set a system of $d_{m}$ linearly independent functions. Then by applying the GramSchmidt process, we get the desired orthonormal basis. Now (b) follows immediately from (a).

(c) The equation

$\left(f, R(g, m) Z_{s g}^{m}\right)_{S}=\left(R\left(g^{-1}, m\right) f, Z_{s g}^{m}\right)_{S}=\left(R\left(g^{-1}, m\right) f\right)(s g)=f(s)=\left(f, Z_{s}^{m}\right)_{S}$, 
which holds for all $f \in H\left(E_{0}, m\right)$, shows immediately that $R(g, m) Z_{s g}^{m}=Z_{s}^{m}$.

Let

$$
K_{0}=\left\{\left[\begin{array}{ccc}
1_{n} & \vdots & 0 \\
\cdots & \vdots & \ldots \\
0 & \vdots & u
\end{array}\right]: u \in S O(k-n)\right\}
$$

then $K_{0}$ is the subgroup of $G_{0}$ fixing I. By Lemma 4.11, we have $R_{0}(u, m) Z_{\mathrm{I} u}^{m}$ $=R_{0}(u, m) Z_{\mathrm{I}}^{m}=Z_{\mathrm{I}}^{m}$ for all $u \in K_{0}$. Let

$$
W_{m}(\mathrm{I})=\left\{f \in H\left(E_{0}, m\right): R_{0}(u, m) f=f, \forall u \in K_{0}\right\} ;
$$

then by Proposition 4.5 and Note 4.7 we deduce that $\operatorname{dim} W_{m}(\mathrm{I})=1$. Since $\mathrm{Z}_{\mathbf{I}}^{m}$ is nonzero and $Z_{\mathbf{I}}^{m}$ belongs to $W_{m}(\mathbf{I})$, we infer that $W_{m}(\mathbf{I})=\left\{c Z_{\mathbf{I}}^{m}: c \in \mathbf{C}\right\}$.

Definition 4.13. For $s_{1} \in S^{n, k}$ we define a parallel on $S^{n, k}$ with respect to the pole $s_{1}$ as the orbit of an $s \in S^{n, k}$ under the subgroup of $G_{0}$ fixing $s_{1}$.

Note that a parallel on $S^{n, k}$ with respect to the pole $I$ is the orbit of some $s \in S^{n, k}$ under $K_{0}$. Now for any $s_{1} \in S^{n, k}, s_{1}=\mathrm{I} g_{1}$ for some $g_{1} \in G_{0}$; hence, the fixing subgroup of $s_{1}$ is $g_{1}^{-1} K_{0} g_{1}$.

Proposition 4.14. Suppose that $s_{1} \in S^{n, k}$; then $f \in H\left(E_{0}, m\right)$ is constant on parallels of $S^{n, k}$ with respect to the pole $s_{1}$ if and only if there exists a constant $C_{1}$ such that $f=C_{1} Z_{s_{1}}^{m}$.

Proof. First observe that $Z_{\mathbf{I}}^{m}$ is constant on parallels with respect to the pole I since for all $s \in S^{n, k}$ and all $u \in K_{0}, Z_{\mathrm{I}}^{m}(s u)=Z_{\mathrm{I} u-1}(s)=Z_{\mathrm{I}}^{m}(s)$. Now for $s_{1} \in S^{n, k}$ such that $s_{1}=I g_{1}$, we have

$$
Z_{s_{1}}^{m}\left(s\left(g_{1}^{-1} u g_{1}\right)\right)=Z_{s_{1} g_{1}^{-1}}\left(s g_{1}^{-1} u\right)=Z_{1}^{m}\left(s g_{1}^{-1}\right)=Z_{s_{1}}^{m}(s) .
$$

Hence, $Z_{s_{1}}^{m}$ is constant on parallels with respect to the pole $s_{1}$. Suppose now that $f \in H\left(E_{0}, m\right)$ is constant on parallels with respect to the pole $s_{1}$, i.e. $f\left(s\left(g_{1}^{-1} u g_{1}\right)\right)$ $=f(s)$ for all $u \in K_{0}$. But this is satisfied if and only if $R(u)\left(R\left(g_{1}\right) f\right)=R\left(g_{1}\right) f$ for all $u \in K_{0}$. Now since $\operatorname{dim} W_{m}(\mathrm{I})=1$, we get $R\left(g_{1}\right) f=C_{1} Z_{\mathbf{I}}^{m}$ for some constant $C_{1} \in \mathrm{C}$. From this we deduce that $f=C_{1} Z_{1 g_{1}}^{m}=C_{1} Z_{s_{1}}^{m}$. and that

PROPOSITION 4.15. Suppose that $f_{s_{1}}\left(s_{2}\right)$ is defined for all $s_{1}, s_{2} \in S^{n, k}$

(a) $f_{s_{1}} \in H\left(E_{0}, m\right)$ for each $s_{1} \in S^{n, k}$,

(b) $R(g) f_{s g}=f_{s}$ for all $s \in S^{n, k}$, and all $g \in G_{0}$.

Then there exists a constant $C$ such that $f_{s_{1}}\left(s_{2}\right)=C Z_{s_{1}}^{m}\left(s_{2}\right)$ for all $s_{1}, s_{2} \in S^{n, k}$.

Proof. Fix $s_{1} \in S^{n, k}$ and let $g$ be an element of the subgroup of $G_{0}$ fix- 
ing $s_{1}$, then from (b) we see that $f_{s_{1}}\left(s_{2} g\right)=f_{s_{1} g}\left(s_{2} g\right)=f_{s_{1}}\left(s_{2}\right)$; therefore by Proposition $4.14 f_{s_{1}}=C\left(s_{1}\right) Z_{s_{1}}^{m}$. To complete the proof of the proposition it suffices to show that $C\left(s_{1}\right)=C\left(s_{2}\right)$ for all $s_{1}, s_{2}$. Let $g_{1}$ be such that $s_{1} g_{1}=$ $s_{2}$; then for all $s \in S^{n, k}$ we have

$$
C\left(s_{2}\right) Z_{s_{1}}^{m}(s)=C\left(s_{2}\right) Z_{s_{2}}^{m}\left(s g_{1}\right)=f_{s_{2}}\left(s g_{1}\right)=f_{s_{1}}(s)=C\left(s_{1}\right) Z_{s_{1}}^{m}(s) \text {. }
$$

From this we deduce that $C\left(s_{1}\right)=C\left(s_{2}\right)$.

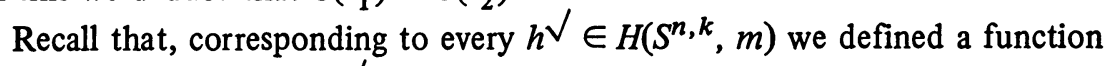
$h$ on $E_{0}$ by setting $h(X)=h^{\sqrt{ }}(s[X])$ for all $X=r[X] s[X]$ with $\operatorname{rank}(X)=n$.

THEOREM 4.16. For every function in $L^{2}\left(E_{0}\right)$ of the form $f h$, where $f$ is

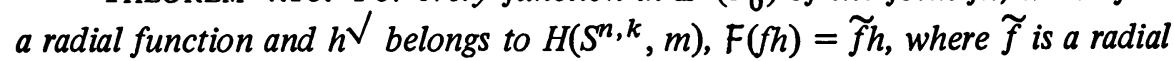
function determined by $f$.

Proof. As usual, it is sufficient to prove the theorem for the case $f h \in$ $L^{1}\left(E_{0}\right) \cap L^{2}\left(E_{0}\right)$. If $X=p^{1 / 2} \sigma$ and $(X \mid Y)=\operatorname{tr}\left(X Y^{t}\right)$, then

$$
\begin{aligned}
{[F(f h)](X) } & =\int_{E_{0}} \exp (-2 \pi i(X \mid Y)) f(Y) h(Y) d Y \\
& =\int_{P_{n}} \int_{S^{n, k}} \exp \left(-2 \pi i\left(p^{1 / 2} \sigma \mid r^{1 / 2} s\right)\right) f^{V}(r) h^{\sqrt{ }}(s)|r|^{1 / 2(k-n-1)} d s d r \\
& =\int_{P_{n}}|r|^{1 / 2(k-n-1)} f(r)\left\{\int_{S^{n, k}} \exp \left(-2 \pi i\left(p^{1 / 2} \sigma \mid r^{1 / 2} s\right)\right) h^{\sqrt{ }}(s) d s\right\} d r .
\end{aligned}
$$

Now

$$
\begin{aligned}
\int_{S^{n, k}} \exp & \left(-2 \pi i\left(p^{1 / 2} \sigma \mid r^{1 / 2} s\right)\right) h^{\sqrt{ }}(s) d s \\
& =\int_{S^{n, k}} \exp \left(-2 \pi i\left(p^{1 / 2} \sigma \mid r^{1 / 2} s\right)\right)\left(\int_{S^{n, k}} h^{\sqrt{ }}(t) \overline{Z_{s}^{m}(t)} d t\right) d s \\
& =\int_{S^{n, k}} h^{\sqrt{ }(t)}\left\{\int_{S^{n, k}} \exp \left(-2 \pi i\left(p^{1 / 2} \sigma \mid r^{1 / 2} s\right)\right) Z_{t}^{m}(s) d s\right\} d t
\end{aligned}
$$

because $Z_{s}^{m}(t)=Z_{t}^{m}(s)$ and $Z_{s}^{m}$ is real valued. Set

$$
F_{\sigma}(t)=\int_{S^{n, k}} \exp \left(-2 \pi i\left(p^{1 / 2} \sigma \mid r^{1 / 2} s\right)\right) Z_{t}^{m}(s) d s .
$$

Then by an argument involving the Fubini theorem and Theorem 4.6, we infer that $F_{\sigma}$ belongs to $H\left(S^{n, k}, m\right)$.

Next

$$
F_{\sigma g}(t g)=\int_{S^{n, k}} \exp \left(-2 \pi i\left(p^{1 / 2} \sigma g \mid r^{1 / 2} s\right)\right) Z_{t g}^{m}(s) d s .
$$

Let $s^{\prime}=s g^{-1}$. Then

$$
\begin{aligned}
F_{\sigma g}(t g) & =\int_{S^{n, k}} \exp \left(-2 \pi i\left(p^{1 / 2} \sigma g \mid r^{1 / 2} s^{\prime} g\right)\right) Z_{t g}^{m}\left(s^{\prime} g\right) d s^{\prime} \\
& =\int_{S^{n, k}} \exp \left(-2 \pi i\left(p^{1 / 2} \sigma \mid r^{1 / 2} s^{\prime}\right)\right) Z_{t}^{m}\left(s^{\prime}\right) d s^{\prime}=F_{\sigma}(t)
\end{aligned}
$$


Therefore, by Proposition 4.15, $F_{\sigma}=C(p, r) Z_{\sigma}^{m}$, where $C(p, r)$ is a constant depending on $p$ and $r$. Now from equation (4.16) we get

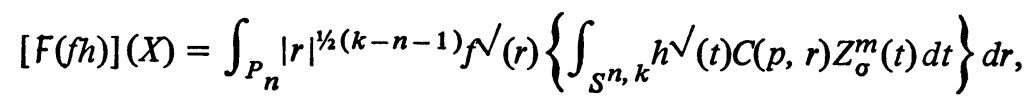

but

$$
\int_{S^{n, k}} h^{\sqrt{ }(t) Z_{\sigma}^{m}(t) d t=h^{\sqrt{ }}(\sigma)}
$$

Hence

$$
[F(f h)](X)=\left\{\int_{P_{n}} f^{V}(r) C(p, r)|r|^{1 / 2(k-n-1)} d r\right\} h(X)
$$

Setting

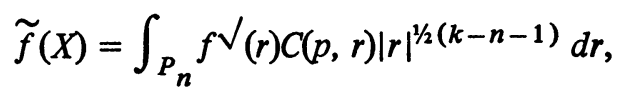

we obtain $F(f h)=\tilde{f h}$.

Note that an explicit expression for $\tilde{f}$ may be obtained in terms of a "generalized Hankel transform" of $f$ (cf. [13, Theorem 3.4, p. 492]).

\section{REFERENCES}

1. A. Borel, Linear algebraic groups, Math. Lecture Notes Ser., Benjamin, New York and Amsterdam, 1969. MR 40 \#4273.

2. A. Borel and A. Weil, Séminaire Bourbaki 6ième année: 1953/54, Exposé 100 par J.-P. Serre: Répresentations linéaires et espaces homogènes Kähleriens des groupes de Lie compacts, Secrétariat mathématique, Paris, 1959. MR 28 \#1087.

3. R. Bott, Homogeneous vector bundles, Ann. of Math. (2) 66 (1957), 203-248. MR 19, 681 .

4. E. Cartan, Leçons sur la géométrie projective complexe, 2ième éd., Gauthier-Villars, Paris, 1950. MR 12, 849.

5. C. Chevalley, Theory of Lie groups. Vol. I, Princeton Math. Ser., vol. 8, Princeton Univ. Press. Princeton, N.J., 1946. MR 7, 412.

6. R. Coifman and G. Weiss, Analyse harmonique non-commutative sur certains espaces homogènes, Lecture Notes in Math., vol. 242, Springer-Verlag, Berlin and New York, 1971.

7. S. Gelbart, $A$ theory of Stiefel harmonics, Trans. Amer. Math. Soc. 192 (1974), 29-50.

8. K. I. Gross and R. A. Kunze, Fourier decompositions of certain representations, Symmetric Spaces, Dekker, New York, 1972, pp. 119-139.

9. - Bessel functions and representation theory. I (in preparation).

10. Harish-Chandra, Differential operators on a semisimple Lie algebra, Amer. J. Math. 79 (1957), 87-120. MR 18, 809.

11. S. Helgason, Differential geometry and symmetric spaces, Pure and Appl. Math., vol. 12, Academic Press, New York, 1962. MR 26 \#2986.

12. - Invariants and fundamental functions, Acta Math. 109 (1963), 241-258. MR 29 \#3581.

13. C. S. Herz, Bessel functions of matrix argument, Ann. of Math. (2) 61 (1955), 474-523. MR 16, 1107.

14. K. Hoffman and R. Kunze, Linear algebra, 2nd ed., Prentice-Hall, Englewood Cliffs, N. J., 1971. MR 43 \#1998. 
15. B. Kostant, Lie group representations on polynomial rings, Amer. J. Math. 85 (1963), 327-404. MR 28 \#1252.

16. Lie algebra cohomology and the generalized Borel-Weil theorem, Ann. of Math. (2) 74 (1961), 329-387. MR 26 \#265.

17. D. Levine, Systems of singular integral operators on spheres, Trans. Amer. Math. Soc. 144 (1969), 493-522.

18. H. Maass, Zur Theorie der harmonischen Formen, Math. Ann. 137 (1959), 142-

149. MR 22 \#12250.

19. - Spherical functions and quadratic forms, J. Indian Math. Soc. 20 (1956),

117-162. MR 19, 252.

20. M. A. Naĭmark, Normed rings, 2nd rev. ed., "Nauka", Moscow, 1968; English transl., Wolters-Noordhoff, Groningen, 1972.

21. E. M. Stein and G. Weiss, Introduction to Fourier analysis on Euclidean spaces, Princeton Math. Ser., no. 32, Princeton Univ. Press, Princeton, N. J., 1971. MR 46 \#4102.

22. R. Strichartz, The explicit Fourier decomposition of $L^{2}(S O(n) / S O(n-m))$, Canad. J. Math. 27 (1975), 294-310.

23. T. Ton-That, Lie group representations and harmonic polynomials of a matrix variable, Ph.D Dissertation, University of California, Irvine, 1974.

24. N. Ja. Vilenkin, Special functions and the theory of group representations, "Nauka", Moscow, 1965; English transl., Transl. Math. Monographs, vol. 22, Amer. Math. Soc., Providence, R. I., 1968. MR 35 \#420; 37 \#5429.

25. N. R. Wallach, Cyclic vectors and irreducibility for principal series representations, Trans. Amer. Math. Soc. 158 (1971), 107-113. MR 43 \#7558.

26. H. Weyl, The classical groups. Their invariants and representations, 2 nd ed., Princeton Univ. Press, Princeton, N. J., 1946.

27. D. P. Želobenko, The theory of linear representations of complex and real Lie groups, Trudy Moskov. Mat. Otšc. 12 (1963), 53-98 = Trans. Moscow Math. Soc. 1963, 57-110. MR $29 \# 2330$.

28. Classical groups. Spectral analysis of finite-dimensional representations, Uspehi Mat. Nauk 17 (1962), no. 1 (103), 27-120 = Russian Math. Surveys 17 (1962), no. 1, 1-94. MR 25 \#129.

\section{DEPARTMENT OF MATHEMATICS, HARVARD UNIVERSITY, CAMBRIDGE, MASSACHUSETTS 02138}

Current address: Department of Mathematics, University of Iowa, Iowa City, Iowa 